\title{
Preliminary Test Estimators and Confidence Intervals for the Parametric Functions of the Moore and Bilikam Family of Lifetime Distributions Based on Records
}

Ajit Chaturvedi University of Delhi

\author{
Suk-Bok Kang \\ Yeungnam University
}

\author{
Ananya Malhotra \\ University of Delhi
}

\begin{abstract}
We consider two measures of reliability functions namely $R(t)=P(X>t)$ and $P=P(X>Y)$ for the Moore and Bilikam (1978) family of lifetime distributions which covers fourteen distributions as specific cases. For record data from this family of distributions, preliminary test estimators (PTEs) and preliminary test confidence interval (PTCI) based on uniformly minimum variance unbiased estimator (UMVUE), maximum likelihood estimator (MLE), empirical Bayes estimator (EBE) are obtained for the parameter. The bias and mean square error (MSE) (exact and asymptotic) of the proposed estimators are derived to study their relative efficiency and through simulation studies we establish that PTEs perform better than ordinary UMVUE, MLE and EBE. We also obtain the coverage probability $(\mathrm{CP})$ and the expected length of the PTCI of the parameter and establish that the confidence intervals based on MLE are more precise. An application of the ordinary preliminary test estimator is also considered. To the best of the knowledge of the authors, no PTEs have been derived for $R(t)$ and $P$ based on records and thus we define improved PTEs based on MLE and UMVUE of $R(t)$ and $P$. A comparative study of different methods of estimation done through simulations establishes that PTEs perform better than ordinary UMVUE and MLE.
\end{abstract}

Keywords: empirical Bayes estimator, Monte Carlo simulation, Moore and Bilikam family of lifetime distribution, preliminary test confidence interval, preliminary test estimator, record values.

\section{Introduction}

In statistical inference, we often come across problems where some prior information on the parameters (often regarded as constraints) is available, which give rise to restricted models. As the name suggests, the estimators obtained from restricted (unrestricted) models are known as restricted (unrestricted) estimators. Naturally, the validity of a restricted estimator will be questionable and hence it is required to perform a preliminary test on the restrictions. Due to past knowledge or experience, the experimenter may be in a position to make an initial guess on some of the parameters of interest. In such cases, we can provide an improved 
estimator by incorporating the prior information on the parameters. The usage of this prior information on some or all of the parameters of a statistical distribution usually leads to an improved inferential study. The efficiency and validity of restricted model analysis are higher over a restricted parametric space induced by the constraints, while the same holds for unrestricted model analysis over the entire parametric space. Thus, an analysis resulting from restricted or unrestricted models may be subject to a loss in efficiency against the validity of constraints while choosing between two inferential techniques. Hence it is wise to adopt restricted estimation procedures when we have sufficient confidence in the prior information. Bancroft (1944) introduced the use of PTEs and eventually further advancements were proposed by Saleh and Sen (1978), Saleh and Kibria (1993),Kibria (2004), Saleh (2006), Kibria and Saleh (1993, 2004, 2005, 2006, 2010) and Belaghi, Arashi, and Tabatabaey (2014, 2015).

Until now, in the literature of inferential reliability, the researchers have developed PTEs of the parameters of different distributions. However, to the best of the knowledge of the authors, PTEs are not available for reliability functions $R(t)$ and $P$. In the present paper, we derive PTEs for the powers of the parameter and two measures of reliability functions, namely $R(t)$ and $P$. The reliability function $R(t)$ is defined as the probability of failure-free operation until time $t$. Thus, if the random variable (RV) $X$ denotes the lifetime of an item or a system, then $R(t)=P(X>t)$. Another measure of reliability under stress-strength setup is the probability $P=P(X>Y)$, which represents the reliability of an item or a system of random strength $X$ subject to random stress $Y$. A lot of work has been done in the literature for the point estimation and testing of $R(t)$ and $P$ based on record values. For a brief review, one may refer to Chaturvedi and Malhotra (2017a,b).

Chandler (1952) introduced the concept of record values. Based on records, inferential procedures for the parameters of different distributions have been developed by Glick (1978), Nagaraja (1988a,b), Balakrishnan, Ahsanullah, and Chan (1995), Arnold, Balakrishnan, and Nagaraja (1992), Habibi, Arghami, and Ahmadi (2006), Arashi and Emadi (2008), Razmkhah, Morabbi, and Ahmadi (2012) and others.

Let $X$ be a RV from the Moore and Bilikam (1978) family of lifetime distributions with probability density function $(\mathrm{PDF})$

$$
f(x ; \beta, \theta)=\frac{\beta}{\theta} g^{\prime}(x) g^{\beta-1}(x) e^{-\frac{g^{\beta}(x)}{\theta}} ; \quad x, \beta, \theta>0
$$

and cumulative distribution function $(\mathrm{CDF})$

$$
F(x ; \beta, \theta)=1-e^{-\frac{g^{\beta}(x)}{\theta}} ; \quad x, \beta, \theta>0
$$

Here, $g(x)$ is a real-valued, strictly increasing function of $x$ with $g\left(0^{+}\right)=0, g(\infty)=\infty$, and $g^{\prime}(x)$ denotes the derivative of $g(x)$ with respect to $x$. The PDF in (1.1) representing a family of lifetime distributions covers the following probabilistic models as specific cases

\begin{tabular}{ccc}
\hline I & $\begin{array}{c}\text { Exponential distribution } \\
\text { (Johnson and Kotz (1970), p.166) }\end{array}$ & $\begin{array}{c}g(x)=x \\
\text { and } \beta=1\end{array}$ \\
\hline II & Weibull distribution & $g(x)=x$ \\
& (Johnson and Kotz (1970), p.250) & \\
\hline III & Burr type XII distribution & $g(x)=\log \left(1+x^{b}\right)$ \\
& (Burr (1942), Burr and Cislak (1968)) & $b>0$ and $\beta=1$ \\
\hline IV & Pareto distribution & $g(x)=\log \left(\frac{x}{a}\right)$ \\
& (Johnson and Kotz (1970), p.233) & and $\beta=1$ \\
\hline V & Rayleigh distribution & $g(x)=x$ \\
& (Johnson and Kotz (1970), p.200) & and $\beta=2$ \\
\hline VI & Lomax (1954) distribution & $g(x)=\log \left(1+\frac{x}{\nu}\right)$ \\
& & $\nu>0$ and $\beta=1$ \\
\hline
\end{tabular}




\begin{tabular}{ccc}
\hline VII & Burr distribution with scale parameter $\nu$ & $g(x)=\log \left(1+\frac{x^{b}}{\nu}\right)$ \\
& Tadikamalla (1980) & $b>0, \nu>0$ and $\beta=1$ \\
\hline VIII & modified Weibull distribution of Lai, Xie, and Murthy (2003) & $g(x)=x^{\gamma} e^{\nu x}$ \\
& & $\gamma>0, \nu>0$ and $\beta=1$ \\
\hline IX & generalised Pareto distribution of Ljubo (1965) & $g(x)=(x-a)+\frac{\nu}{\lambda} \log \left(\frac{x+\nu}{a+\lambda}\right)$ \\
& & $\nu>0, \lambda>0$ and $\beta=1$ \\
\hline X & linear exponential distribution & $g(x)=b x+\frac{\lambda}{2} x^{2}$ \\
& Mahmoud and Al-Nagar (2009) & $\lambda>0, b>0$ and $\beta=1$ \\
\hline XI & generalised power Weibull distribution & $g(x)=\left(1+x^{b}\right)^{\lambda}-1$ \\
& Nikulin and Haghighi (2006) & $b>0, \lambda>0$ and $\beta=1$ \\
\hline XII & Gompertz distribution & $g(x)=\frac{\alpha}{b}\left(e^{b x}-1\right)$ \\
& Khan and Zia (2009) & $\alpha>0, b>0$ and $\beta=1$ \\
\hline XIII & Chen (2000) distribution & $g(x)=\left(e^{x^{b}}-1\right)$ \\
& & $b>0$ and $\beta=1$ \\
\hline XIV & two-parameter exponential distribution & $g(x)=(x-a)$ \\
& Ahsanullah (1980) & and $\beta=1$ \\
\hline
\end{tabular}

Let $X_{1}, X_{2}, \ldots$ be an infinite sequence of independent and identically distributed (iid) RVs from (1.1). An observation $X_{j}$ will be called an upper record value (or simply a record) if its value exceeds that of all previous observations. Thus $X_{j}$ is a record if $X_{j}>X_{i}$ for every $i<j$. The record time sequence $T_{n}, n \geq 0$ is defined as

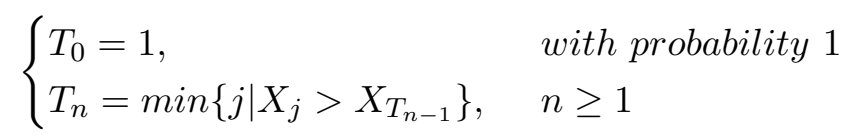

The record value sequence $\left\{R_{n}\right\}$ is then defined as

$$
R_{n}=X_{T_{n}}, n=0,1,2, \ldots
$$

The likelihood function of the first $n+1$ upper record values $R_{0}, R_{1}, R_{2}, \ldots, R_{n}$ is

$$
L\left(\theta \mid R_{0}, R_{1}, R_{2}, \ldots, R_{n}\right)=f\left(R_{n} ; \beta, \theta\right) \prod_{i=0}^{n-1} \frac{f\left(R_{i} ; \beta, \theta\right)}{1-F\left(R_{i} ; \beta, \theta\right)}
$$

Belaghi, Arashi, and Tabatabaey $(2014,2015)$ constructed PTEs and PTCIs based on record values for the Burr XII model. In this paper we construct some PTEs on the basis of records for the powers of the parameter and reliability functions of Moore and Bilikam (1978) family of lifetime distribution which covers as many as fourteen distributions as its specific cases, in two different situations.

In Section 2, we assume that the shape parameter $\beta$ is known and propose three different PTEs for the powers of the scale parameter $\theta$ and the reliability functions $R(t)$ and $P$. Then bias and MSE of the proposed estimators are obtained. We then obtain confidence intervals based on the PTEs of the parameter and hence obtain its CP and expected length (EL). Also the superiority intervals of our new estimators are tabulated. We shall observe that near the null hypothesis, the relative efficiency of PTEs increases and thus the proposed estimators dominate the usual estimators. In Section 3 we consider the case where $\beta$ is unknown while $\theta$ is known. In Section 4, we suppose that both the parameters $\beta$ and $\theta$ are unknown and follow an approach by Gulati and Padgett (1994b,a, 1995). In this case, using asymptotic normality of the MLE, we obtain superiority conditions for the proposed PTE. Also, asymptotic bias, MSE and asymptotic relative efficiency of the proposed PTE of the parameter are obtained. 


\section{Estimation of parametric functions when the shape parameter is known}

The importance of discussion of this case lies in the derivation of UMVUE of parameter $\theta$ with the help of complete and sufficient statistics of the parameter $\theta$ which exists only when the shape parameter $\beta$ is assumed to be known.

\subsection{Proposed preliminary test estimators}

Assuming the shape parameter $\beta$ to be known, it follows from (1.3) that

$$
L\left(\theta \mid R_{0}, R_{1}, R_{2}, \ldots, R_{n}\right)=\left(\frac{\beta}{\theta}\right)^{n+1} \exp \left(\frac{-g^{\beta}\left(R_{n}\right)}{\theta}\right) \prod_{i=0}^{n} g^{\prime}\left(R_{i}\right) g^{\beta-1}\left(R_{i}\right)
$$

We now estimate the powers of the parameter. The reason for estimating the powers of the parameter being that it takes place in the expressions for the moments and hazard rate of the distribution. Thus by estimating the powers of parameter, one can easily develop PTEs for the moments and hazard rate. Then, for $p \in(-\infty, \infty), p \neq 0$ the MLE of $\theta^{p}$ is

$$
\hat{\theta}_{M L}^{p}=\left(\frac{g^{\beta}\left(R_{n}\right)}{n+1}\right)^{p}
$$

where $g^{\beta}\left(R_{n}\right)$ is the complete and sufficient statistic of $\theta$ and has gamma distribution with parameters $(n+1, \theta)$. Further, the UMVUE of $\theta^{p}$ is

$$
\hat{\theta}_{U}^{p}=\frac{\Gamma(n+1)}{\Gamma(n+p+1)}\left(g^{\beta}\left(R_{n}\right)\right)^{p}
$$

Now, if we consider conjugate prior distribution of $\theta$ to be inverted-gamma distribution with parameters $(\mu, \nu)$ and

$$
\pi(\theta)=\frac{\mu^{\nu}}{\Gamma(\nu) \theta^{\nu+1}} e^{-\frac{\mu}{\theta}}, \mu, \theta>0 \text { and } \nu \text { is a positive integer }
$$

then the posterior distribution of $\theta$ given $R_{0}, R_{1}, R_{2}, \ldots, R_{n}$ is

$$
\pi\left(\theta \mid R_{0}, R_{1}, R_{2}, \ldots, R_{n}\right)=\frac{\left(g^{\beta}\left(R_{n}\right)+\mu\right)^{n+\nu+1}}{\Gamma(n+\nu+1) \theta^{n+\nu+2}} e^{-\frac{\left(g^{\beta}\left(R_{n}\right)+\mu\right)}{\theta}}
$$

Under squared error loss function, the Bayes estimator of $\theta^{p}$ is

$$
\hat{\theta}_{B}^{p}=\frac{\Gamma(n+\nu-p+1)}{\Gamma(n+\nu+1)}\left(g^{\beta}\left(R_{n}\right)+\mu\right)^{p}, \nu>-(n-p+1)
$$

Also, the marginal distribution of $R_{0}, R_{1}, R_{2}, \ldots, R_{n}$ given $\mu$ and $\nu$ is

$$
\begin{aligned}
m\left(R_{0}, R_{1}, R_{2}, \ldots, R_{n} \mid \mu, \nu\right) & =\int_{0}^{\infty} \pi(\theta) L\left(\beta, \theta \mid R_{0}, R_{1}, R_{2}, \ldots, R_{n}\right) d \theta \\
& =\frac{\mu^{\nu} \Gamma(n+\nu+1)}{\left(g^{\beta}\left(R_{n}\right)+\mu\right)^{n+\nu+1} \Gamma(\nu)} \beta^{n+1} \prod_{i=0}^{n} g^{\beta-1}\left(R_{i}\right) g^{\prime}\left(R_{i}\right)
\end{aligned}
$$

Taking the natural logarithm $l$ of the above marginal distribution, the MLE of $\mu$ and $\nu$ can be obtained from the solution of the following system

$$
\frac{\partial l}{\partial \mu}=\frac{\nu}{\mu}=\frac{n+\nu+1}{g^{\beta}\left(R_{n}\right)+\mu}=0
$$


and

$$
\frac{\partial l}{\partial \nu}=\log (\mu)+\frac{\partial}{\partial \nu}\left[\log \left(\frac{\Gamma(n+\nu+1)}{\Gamma(\nu)}\right)\right]-\log \left(g^{\beta}\left(R_{n}\right)+\mu\right)=0
$$

Denoting the MLE of $\mu$ and $\nu$ by $\hat{\mu}_{M L}$ and $\hat{\nu}_{M L}$ respectively, there exists a relation between them given by

$$
\hat{\mu}_{M L}=\frac{g^{\beta}\left(R_{n}\right) \hat{\nu}_{M L}}{n+1}
$$

Therefore from (2.6), the EBE of $\theta^{p}$ is

$$
\hat{\theta}_{E B}^{p}=\frac{\Gamma\left(n+\hat{\nu}_{M L}-p+1\right)}{\Gamma\left(n+\hat{\nu}_{M L}+1\right)}\left(g^{\beta}\left(R_{n}\right)+\hat{\mu}_{M L}\right)^{p}, \hat{\nu}_{M L}>-(n-p+1)
$$

Sometimes, due to past knowledge or experience, the experimenter may be able to make an initial guess on some of the parameters of interest. This prior information can be expressed in the form of the following hypothesis

$$
\begin{aligned}
& H_{0}: \theta=\theta_{0} \\
& H_{1}: \theta \neq \theta_{0}
\end{aligned}
$$

Then based on classical hypothesis testing, the critical region is given by

$$
\left\{0<g^{\beta}\left(R_{n}\right)<k_{0}\right\} \cup\left\{k_{0}^{\prime}<g^{\beta}\left(R_{n}\right)<\infty\right\}
$$

where $k_{0}$ and $k_{0}^{\prime}$ are obtained such that $k_{0}=\frac{\theta_{0}}{2} \chi_{2(n+1)}^{2}\left(\frac{\alpha}{2}\right), k_{0}^{\prime}=\frac{\theta_{0}}{2} \chi_{2(n+1)}^{2}\left(1-\frac{\alpha}{2}\right)$, and $\alpha$ is the level of significance. Or, equivalently we reject $H_{0}$ if

$$
\frac{2 g^{\beta}\left(R_{n}\right)}{\theta_{0}}<C_{2} \text { or } \frac{2 g^{\beta}\left(R_{n}\right)}{\theta_{0}}>C_{1}
$$

where $C_{1}=\chi_{2(n+1)}^{2}\left(1-\frac{\alpha}{2}\right)$ and $C_{2}=\chi_{2(n+1)}^{2}\left(\frac{\alpha}{2}\right)$.

We can now define the three PTEs for $\theta^{p}$ based on UMVUE, MLE and EBE respectively as

$$
\begin{aligned}
\hat{\theta}_{P T-U}^{p} & =\hat{\theta}_{U}^{p}-\left(\hat{\theta}_{U}^{p}-\theta_{0}^{p}\right) I(A) \\
\hat{\theta}_{P T-M L}^{p} & =\hat{\theta}_{M L}^{p}-\left(\hat{\theta}_{M L}^{p}-\theta_{0}^{p}\right) I(A) \\
\hat{\theta}_{P T-E B}^{p} & =\hat{\theta}_{E B}^{p}-\left(\hat{\theta}_{E B}^{p}-\theta_{0}^{p}\right) I(A)
\end{aligned}
$$

where $I(A)$ is the indicator function of the set

$$
A=\left\{\chi_{2(n+1)}^{2} \mid C_{2} \leq \chi_{2(n+1)}^{2} \leq C_{1}\right\}
$$

The MLE and UMVUE of $R(t)$ are respectively given by

$$
\hat{R}(t)=\exp \left\{\frac{-(n+1) g^{\beta}(t)}{g^{\beta}\left(R_{n}\right)}\right\}
$$

and

$$
\tilde{R}(t)= \begin{cases}{\left[1-\frac{g^{\beta}(t)}{g^{\beta}\left(R_{n}\right)}\right]^{n},} & g^{\beta}(t)<g^{\beta}\left(R_{n}\right) \\ 0, & \text { otherwise }\end{cases}
$$

Thus, we define two different PTEs of $R(t)$ based on MLE and UMVUE as follows:

$$
\begin{aligned}
\hat{R}(t)_{P T-M L} & =\hat{R}(t)-\left(\hat{R}(t)-R_{0}(t)\right) I(A) \\
\tilde{R}(t)_{P T-U} & =\tilde{R}(t)-\left(\tilde{R}(t)-R_{0}(t)\right) I(A)
\end{aligned}
$$


where $R_{0}(t)=\exp \left(-\frac{g^{\beta}(t)}{\theta_{0}}\right)$ is the hypothesized value of $R(t)$.

Let $X$ and $Y$ be two independent RVs from the same family of lifetime distributions with parameters $\theta_{1}$ and $\theta_{2}$ respectively and $\beta_{1}=\beta_{2}$. Let $R_{0}, R_{1}, \ldots, R_{n}$ be $n+1$ record values from distribution of $X$ and $R_{0}^{*}, R_{1}^{*}, \ldots, R_{m}^{*}$ be $m+1$ records from distribution of $Y$. Then, $P=\frac{\theta_{1}}{\theta_{1}+\theta_{2}}$. Suppose we want to test

$$
\begin{aligned}
& H_{0}: P=P_{0} \\
& H_{1}: P \neq P_{0}
\end{aligned}
$$

Note that $H_{0}$ is equivalent to $\theta_{1}=k \theta_{2}$ where $k=\frac{P_{0}}{1-P_{0}}$. Thus, $H_{0}: \theta_{1}=k \theta_{2}$ and $H_{1}: \theta_{1} \neq$ $k \theta_{2}$. Since $g^{\beta}\left(R_{n}\right)$ and $g^{\beta}\left(R_{m}^{*}\right)$ follow gamma distribution with parameters $\left(n+1, \theta_{1}\right)$ and $\left(m+1, \theta_{2}\right)$ respectively, it is easy to see that $\frac{(m+1) \theta_{2} g^{\beta}\left(R_{n}\right)}{(n+1) \theta_{1} g^{\beta}\left(R_{m}^{*}\right)} \sim F_{2(n+1), 2(m+1)}$ and the critical region is given by

$$
\left\{\frac{g^{\beta}\left(R_{n}\right)}{g^{\beta}\left(R_{m}^{*}\right)}<k_{1}\right\} \cup\left\{\frac{g^{\beta}\left(R_{n}\right)}{g^{\beta}\left(R_{m}^{*}\right)}>k_{1}^{\prime}\right\}
$$

where $k_{1}=\frac{k(n+1)}{(m+1)} F_{2(n+1), 2(m+1)}\left(\frac{\alpha}{2}\right)$ and $k_{1}^{\prime}=\frac{k(n+1)}{(m+1)} F_{2(n+1), 2(m+1)}\left(1-\frac{\alpha}{2}\right)$. Thus, we define two PTEs of $P$ based on MLE and UMVUE of $P$ as follows:

$$
\begin{aligned}
\hat{P}_{P T-M L} & =\hat{P}-\left(\hat{P}-P_{0}\right) I(B) \\
\tilde{P}_{P T-U} & =\tilde{P}-\left(\tilde{P}-P_{0}\right) I(B)
\end{aligned}
$$

where $I(B)$ is the indicator function of the set

$$
B=\left\{F_{2(n+1), 2(m+1)} \mid C_{4}<F_{2(n+1), 2(m+1)}<C_{3}\right\}
$$

Here, $C_{3}=F_{2(n+1), 2(m+1)}\left(1-\frac{\alpha}{2}\right), C_{4}=F_{2(n+1), 2(m+1)}\left(\frac{\alpha}{2}\right)$ and $\hat{P}$ and $\tilde{P}$ are the MLE and UMVUE of $P$ respectively defined as

$$
\hat{P}=\frac{(m+1) g^{\beta}\left(R_{n}\right)}{(m+1) g^{\beta}\left(R_{n}\right)+(n+1) g^{\beta}\left(R_{m}^{*}\right)}
$$

and

$$
\tilde{P}= \begin{cases}\sum_{i=0}^{m-1} \frac{(-1)^{i} m ! n !}{(m-i-1) !(n+i+1) !}\left\{\frac{g^{\beta}\left(R_{n}\right)}{g^{\beta}\left(R_{m}^{*}\right)}\right\}^{i+1}, & g^{\beta}\left(R_{n}\right)<g^{\beta}\left(R_{m}^{*}\right) \\ \sum_{i=0}^{n} \frac{(-1)^{i} m ! n !}{(n-i) !(m+i) !}\left\{\frac{g^{\beta}\left(R_{m}^{*}\right)}{g^{\beta}\left(R_{n}\right)}\right\}^{i}, & g^{\beta}\left(R_{m}^{*}\right)<g^{\beta}\left(R_{n}\right)\end{cases}
$$

The expressions for MLE and UMVUE of $R(t)$ and $P$ can be derived on the same lines as the proofs of theorems 2, 5,6 and 9 in Chaturvedi and Malhotra (2017a).

\subsection{Bias and mean square error}

In this section we derive bias and MSE of PTEs based on UMVUE, MLE and EBE. Assuming $\lambda=\frac{\theta_{0}}{\theta}$, we have

$$
\begin{aligned}
\operatorname{Bias}\left(\hat{\theta}_{P T-U}^{p}\right)= & E\left[\hat{\theta}_{U}^{p}-\left(\hat{\theta}_{U}^{p}-\theta_{0}^{p}\right) I(A)-\theta^{p}\right] \\
= & \theta_{0}^{p}\left[\left\{H_{2 n+2}\left(\lambda C_{1}\right)-H_{2 n+2}\left(\lambda C_{2}\right)\right\}\right. \\
& \left.-\left(\frac{1}{\lambda}\right)^{p}\left\{H_{2(n+p+1)}\left(\lambda C_{1}\right)-H_{2(n+p+1)}\left(\lambda C_{2}\right)\right\}\right]
\end{aligned}
$$

where $H_{\gamma}($.$) stands for the CDF of \chi^{2}$ distribution with $\gamma$ degrees of freedom. 
Since

$$
\begin{aligned}
\operatorname{Var}\left(\hat{\theta}_{P T-U}^{p}\right)= & \operatorname{Var}\left(\hat{\theta}_{U}^{p}\right)+\operatorname{Var}\left(\left(\hat{\theta}_{U}^{p}-\theta_{0}^{p}\right) I(A)\right)-2 \operatorname{Cov}\left(\hat{\theta}_{U}^{p},\left(\hat{\theta}_{U}^{p}-\theta_{0}^{p}\right) I(A)\right) \\
= & \theta^{2 p}\left[\frac{\Gamma(n+2 p+1) \Gamma(n+1)}{\Gamma^{2}(n+p+1)}-1\right] \\
& +\theta^{2 p} \frac{\Gamma(n+2 p+1) \Gamma(n+1)}{\Gamma^{2}(n+p+1)}\left\{H_{2(n+2 p+1)}\left(\lambda C_{1}\right)-H_{2(n+2 p+1)}\left(\lambda C_{2}\right)\right\} \\
& -\theta^{2 p}\left\{H_{2(n+p+1)}\left(\lambda C_{1}\right)-H_{2(n+p+1)}\left(\lambda C_{2}\right)\right\}^{2} \\
& +\theta_{0}^{2 p}\left\{H_{2 n+2}\left(\lambda C_{1}\right)-H_{2 n+2}\left(\lambda C_{2}\right)\right\}\left[1-\left\{H_{2 n+2}\left(\lambda C_{1}\right)-H_{2 n+2}\left(\lambda C_{2}\right)\right\}\right] \\
& -2 \theta_{0}^{p} \theta^{p}\left\{H_{2(n+p+1)}\left(\lambda C_{1}\right)-H_{2(n+p+1)}\left(\lambda C_{2}\right)\right\} \\
& \times\left[1-\left\{H_{2 n+2}\left(\lambda C_{1}\right)-H_{2 n+2}\left(\lambda C_{2}\right)\right\}\right] \\
& -2 \theta^{2 p} \frac{\Gamma(n+2 p+1) \Gamma(n+1)}{\Gamma^{2}(n+p+1)}\left\{H_{2(n+2 p+1)}\left(\lambda C_{1}\right)-H_{2(n+2 p+1)}\left(\lambda C_{2}\right)\right\} \\
& +2 \theta_{0}^{p} \theta^{p}\left\{H_{2(n+p+1)}\left(\lambda C_{1}\right)-H_{2(n+p+1)}\left(\lambda C_{2}\right)\right\} \\
& +2 \theta^{2 p}\left\{H_{2(n+p+1)}\left(\lambda C_{1}\right)-H_{2(n+p+1)}\left(\lambda C_{2}\right)\right\} \\
& -2 \theta^{p} \theta_{0}^{p}\left\{H_{2 n+2}\left(\lambda C_{1}\right)-H_{2 n+2}\left(\lambda C_{2}\right)\right\},
\end{aligned}
$$

the MSE of PTE of $\theta^{p}$ based on UMVUE can be simplified to be

$$
\begin{aligned}
M S E\left(\hat{\theta}_{P T-U}^{p}\right)= & \theta^{2 p}\left[\frac{\Gamma(n+2 p+1) \Gamma(n+1)}{\Gamma^{2}(n+p+1)}-1\right] \\
& -\theta^{2 p} \frac{\Gamma(n+2 p+1) \Gamma(n+1)}{\Gamma^{2}(n+p+1)} \\
& \times\left\{H_{2(n+2 p+1)}\left(\lambda C_{1}\right)-H_{2(n+2 p+1)}\left(\lambda C_{2}\right)\right\} \\
& +\left(\theta_{0}^{2 p}-2 \theta^{p} \theta_{0}^{p}\right)\left\{H_{2 n+2}\left(\lambda C_{1}\right)-H_{2 n+2}\left(\lambda C_{2}\right)\right\} \\
& +2 \theta^{2 p}\left\{H_{2(n+p+1)}\left(\lambda C_{1}\right)-H_{2(n+p+1)}\left(\lambda C_{2}\right)\right\}
\end{aligned}
$$

Now, the bias of PTE of $\theta^{p}$ based on MLE is

$$
\begin{aligned}
\operatorname{Bias}\left(\hat{\theta}_{P T-U}^{p}\right)= & \left(\frac{\theta}{n+1}\right)^{p} \frac{\Gamma(n+p+1)}{\Gamma(n+1)} \\
& \times\left[1-\left\{H_{2(n+p+1)}\left(\lambda C_{1}\right)-H_{2(n+p+1)}\left(\lambda C_{2}\right)\right\}\right] \\
& +\theta_{0}^{p}\left\{H_{2 n+2}\left(\lambda C_{1}\right)-H_{2 n+2}\left(\lambda C_{2}\right)\right\}-\theta^{p}
\end{aligned}
$$

Then the MSE of PTE of $\theta^{p}$ based on MLE is obtained as

$$
\begin{aligned}
M S E\left(\hat{\theta}_{P T-M L}^{p}\right)= & \left\{\left(\frac{\theta}{n+1}\right)^{p} \frac{\Gamma(n+p+1)}{\Gamma(n+1)}\right\}^{2}\left[\frac{\Gamma(n+2 p+1) \Gamma(n+1)}{\Gamma^{2}(n+p+1)}-1\right] \\
& -\left(\frac{\theta}{n+1}\right)^{2 p} \frac{\Gamma(n+2 p+1)}{\Gamma(n+1)}\left\{H_{2(n+2 p+1)}\left(\lambda C_{1}\right)-H_{2(n+2 p+1)}\left(\lambda C_{2}\right)\right\} \\
& -\left\{\left(\frac{\theta}{n+1}\right)^{p} \frac{\Gamma(n+p+1)}{\Gamma(n+1)}\right\}^{2}\left\{H_{2(n+p+1)}\left(\lambda C_{1}\right)-H_{2(n+p+1)}\left(\lambda C_{2}\right)\right\}^{2} \\
& +\theta_{0}^{2 p}\left\{H_{2 n+2}\left(\lambda C_{1}\right)-H_{2 n+2}\left(\lambda C_{2}\right)\right\}\left[1-\left\{H_{2 n+2}\left(\lambda C_{1}\right)-H_{2 n+2}\left(\lambda C_{2}\right)\right\}\right] \\
& -2 \theta_{0}^{p}\left(\frac{\theta}{n+1}\right)^{p} \frac{\Gamma(n+p+1)}{\Gamma(n+1)}\left\{H_{2(n+p+1)}\left(\lambda C_{1}\right)-H_{2(n+p+1)}\left(\lambda C_{2}\right)\right\}
\end{aligned}
$$




$$
\begin{aligned}
& \times\left[1-\left\{H_{2 n+2}\left(\lambda C_{1}\right)-H_{2 n+2}\left(\lambda C_{2}\right)\right\}\right] \\
& +2 \theta_{0}^{p}\left(\frac{\theta}{n+1}\right)^{p} \frac{\Gamma(n+p+1)}{\Gamma(n+1)}\left[\left\{H_{2(n+p+1)}\left(\lambda C_{1}\right)-H_{2(n+p+1)}\left(\lambda C_{2}\right)\right\}\right. \\
& \left.-\left\{H_{2 n+2}\left(\lambda C_{1}\right)-H_{2 n+2}\left(\lambda C_{2}\right)\right\}\right] \\
& +2\left\{\left(\frac{\theta}{n+1}\right)^{p} \frac{\Gamma(n+p+1)}{\Gamma(n+1)}\right\}^{2}\left\{H_{2(n+p+1)}\left(\lambda C_{1}\right)-H_{2(n+p+1)}\left(\lambda C_{2}\right)\right\} \\
& +\left[\left(\frac{\theta}{n+1}\right)^{p} \frac{\Gamma(n+p+1)}{\Gamma(n+1)}\left[1-\left\{H_{2(n+p+1)}\left(\lambda C_{1}\right)-H_{2(n+p+1)}\left(\lambda C_{2}\right)\right\}\right]\right. \\
& \left.+\theta_{0}^{p}\left\{H_{2 n+2}\left(\lambda C_{1}\right)-H_{2 n+2}\left(\lambda C_{2}\right)\right\}-\theta^{p}\right]^{2}
\end{aligned}
$$

Before deriving bias and MSE for PTE of $\theta^{p}$ based on EBE, for the sake of simplicity we define the following:

$$
\begin{gathered}
\varphi_{1}=\int_{0}^{\infty}\left(y+\hat{\mu}_{M L}\right)^{p} y^{n} e^{-\frac{y}{\theta}} d y, \quad \varphi_{2}=\int_{0}^{\infty}\left(y+\hat{\mu}_{M L}\right)^{2 p} y^{n} e^{-\frac{y}{\theta}} d y \\
\varphi_{3}=\int_{\frac{\theta_{0} C_{2}}{2}}^{\frac{\theta_{0} C_{1}}{2}}\left(y+\hat{\mu}_{M L}\right)^{p} y^{n} e^{-\frac{y}{\theta}} d y, \quad \varphi_{4}=\int_{\frac{\theta_{0} C_{2}}{2}}^{\frac{\theta_{0} C_{1}}{2}}\left(y+\hat{\mu}_{M L}\right)^{2 p} y^{n} e^{-\frac{y}{\theta}} d y
\end{gathered}
$$

Therefore, we have

$$
\begin{aligned}
\operatorname{Bias}\left(\hat{\theta}_{P T-E B}^{p}\right)= & \frac{\Gamma\left(n+\hat{\nu}_{M L}-p+1\right)}{\Gamma\left(n+\hat{\nu}_{M L}+1\right) \Gamma(n+1) \theta^{n+1}}\left(\varphi_{1}-\varphi_{3}\right) \\
& +\theta_{0}^{p}\left\{H_{2 n+2}\left(\lambda C_{1}\right)-H_{2 n+2}\left(\lambda C_{2}\right)\right\}-\theta^{p}
\end{aligned}
$$

and

$$
\begin{aligned}
M S E\left(\hat{\theta}_{P T-E B}^{p}\right)= & \left\{\frac{\Gamma\left(n+\hat{\nu}_{M L}-p+1\right)}{\Gamma\left(n+\hat{\nu}_{M L}+1\right)}\right\}^{2} \frac{\left(\varphi_{2}-\varphi_{4}\right)}{\Gamma(n+1) \theta^{n+1}} \\
& -\left[\frac{\Gamma\left(n+\hat{\nu}_{M L}-p+1\right)}{\Gamma\left(n+\hat{\nu}_{M L}+1\right) \Gamma(n+1) \theta^{n+1}}\right]^{2}\left(\varphi_{1}-\varphi_{3}\right)^{2} \\
& +\theta_{0}^{2 p}\left\{H_{2 n+2}\left(\lambda C_{1}\right)-H_{2 n+2}\left(\lambda C_{2}\right)\right\}\left[1-\left\{H_{2 n+2}\left(\lambda C_{1}\right)-H_{2 n+2}\left(\lambda C_{2}\right)\right\}\right] \\
& +2 \theta_{0}^{p} \frac{\Gamma\left(n+\hat{\nu}_{M L}-p+1\right)}{\Gamma\left(n+\hat{\nu}_{M L}+1\right) \Gamma(n+1) \theta^{n+1}} \\
& \times\left(\varphi_{3}-\varphi_{1}\right)\left\{H_{2 n+2}\left(\lambda C_{1}\right)-H_{2 n+2}\left(\lambda C_{2}\right)\right\} \\
& +\left[\frac{\Gamma\left(n+\hat{\nu}_{M L}-p+1\right)}{\Gamma\left(n+\hat{\nu}_{M L}+1\right) \Gamma(n+1) \theta^{n+1}}\left(\varphi_{1}-\varphi_{3}\right)\right. \\
& \left.+\theta_{0}^{p}\left\{H_{2 n+2}\left(\lambda C_{1}\right)-H_{2 n+2}\left(\lambda C_{2}\right)\right\}-\theta^{p}\right]^{2}
\end{aligned}
$$

Now, we derive bias and MSE expressions of PTEs of $R(t)$ based on MLE and UMVUE.

For the sake of simplicity, we define the following:

$$
\begin{aligned}
& \varphi_{5}=\int_{\frac{C_{2}}{2}}^{\frac{C_{1}}{2}} \frac{z^{n}}{n !} \exp \left[-\left(z+\frac{(n+1) g^{\beta}(t)}{\theta z}\right)\right] d z, \\
& \varphi_{6}=\int_{\frac{C_{2}}{2}}^{\frac{C_{1}}{2}} \frac{z^{n}}{n !} \exp \left[-\left(z+\frac{2(n+1) g^{\beta}(t)}{\theta z}\right)\right] d z
\end{aligned}
$$

Then, the bias of PTE of $R(t)$ based on MLE is as follows:

$$
\begin{aligned}
\operatorname{Bias}\left(\hat{R}(t)_{P T-M L}\right)= & \frac{1}{\Gamma(n+1)} \int_{0}^{\infty} \exp \left[-\left\{y+\frac{(n+1) g^{\beta}(t)}{\theta y}\right\}\right] y^{n} d y \\
& -\varphi_{5}+R_{0}(t)\left\{H_{2 n+2}\left(\lambda C_{1}\right)-H_{2 n+2}\left(\lambda C_{2}\right)\right\}-R(t)
\end{aligned}
$$


Applying the result of Watson (1952) given by

$$
\int_{0}^{\infty} u^{-m} \exp \left\{-\left(a u+\frac{b}{u}\right)\right\} d u=2\left(\frac{a}{b}\right)^{\frac{m-1}{2}} K_{m-1}(2 \sqrt{a b})
$$

[it is to be noted that $K_{-m}(\cdot)=K_{m}(\cdot)$ for $m=0,1,2, \ldots$ ], we obtain from $(2.25)$ that

$$
\begin{aligned}
\operatorname{Bias}\left(\hat{R}(t)_{P T-M L}\right)= & \frac{2}{n !}\left\{\frac{(n+1) g^{\beta}(t)}{\theta}\right\}^{\frac{n+1}{2}} K_{n+1}\left(2 \sqrt{\frac{(n+1) g^{\beta}(t)}{\theta}}\right) \\
& -\varphi_{5}+R_{0}(t)\left\{H_{2 n+2}\left(\lambda C_{1}\right)-H_{2 n+2}\left(\lambda C_{2}\right)\right\}-R(t)
\end{aligned}
$$

Following which, the MSE of PTE of $R(t)$ based on MLE is

$$
\begin{aligned}
M S E\left(\hat{R}(t)_{P T-M L}\right)= & \frac{2}{n !}\left\{\frac{2(n+1) g^{\beta}(t)}{\theta}\right\}^{\frac{n+1}{2}} K_{n+1}\left(2 \sqrt{\frac{2(n+1) g^{\beta}(t)}{\theta}}\right) \\
& -\left[\frac{2}{n !}\left\{\frac{(n+1) g^{\beta}(t)}{\theta}\right\}^{\frac{n+1}{2}} K_{n+1}\left(2 \sqrt{\frac{(n+1) g^{\beta}(t)}{\theta}}\right)\right]^{2} \\
& +\varphi_{6}-\varphi_{5}^{2}+R_{0}(t)\left\{H_{2 n+2}\left(\lambda C_{1}\right)-H_{2 n+2}\left(\lambda C_{2}\right)\right\} \\
& \times\left\{1-\left\{H_{2 n+2}\left(\lambda C_{1}\right)-H_{2 n+2}\left(\lambda C_{2}\right)\right\}\right\} \\
& +2\left[\frac{2}{n !}\left\{\frac{(n+1) g^{\beta}(t)}{\theta}\right\}^{\frac{n+1}{2}} K_{n+1}\left(2 \sqrt{\frac{(n+1) g^{\beta}(t)}{\theta}}\right)\right] \varphi_{5} \\
& +2 R_{0}(t)\left\{H_{2 n+2}\left(\lambda C_{1}\right)-H_{2 n+2}\left(\lambda C_{2}\right)\right\} \\
& \times\left(\frac{2}{n !}\left\{\frac{(n+1) g^{\beta}(t)}{\theta}\right\}^{\frac{n+1}{2}} K_{n+1}\left(\sqrt{\frac{(n+1) g^{\beta}(t)}{\theta}}\right)^{-\varphi_{5}}\right) \\
& +\left[\frac{2}{n !}\left\{\frac{(n+1) g^{\beta}(t)}{\theta}\right\}^{\frac{n+1}{2}} K_{n+1}\left(2 \sqrt{\frac{(n+1) g^{\beta}(t)}{\theta}}\right)^{2}\right. \\
& \left.-\varphi_{5}+R_{0}(t)\left\{H_{2 n+2}\left(\lambda C_{1}\right)-H_{2 n+2}\left(\lambda C_{2}\right)\right\}-R(t)\right]^{2}
\end{aligned}
$$

Denoting by

$$
\varphi_{7}=\int_{C_{2}}^{C_{1}}\left(1-\frac{2 g^{\beta}(t)}{\theta u}\right)^{2 n} \frac{u^{n} e^{-\frac{u}{2}}}{2^{n+1} n !} d u \text { and } \varphi_{8}=\int_{C_{2}}^{C_{1}}\left(1-\frac{2 g^{\beta}(t)}{\theta u}\right)^{n} \frac{u^{n} e^{-\frac{u}{2}}}{2^{n+1} n !} d u
$$

then, the bias of PTE of $R(t)$ based on UMVUE is

$$
\operatorname{Bias}\left(\tilde{R}(t)_{P T-U}\right)=R_{0}(t)\left\{H_{2 n+2}\left(\lambda C_{1}\right)-H_{2 n+2}\left(\lambda C_{2}\right)\right\}-\varphi_{8}
$$

We have obtained the variance of UMVUE of $R(t)$ as follows:

$$
\begin{aligned}
\operatorname{Var}\{\tilde{R}(t)\}= & \frac{1}{n !}\left\{\frac{g^{\beta}(t)}{\theta}\right\}^{(n+1)} \exp \left\{-\frac{g^{\beta}(t)}{\theta}\right\}\left[\frac{\theta a_{n}}{g^{\beta}(t)}-a_{n-1} \exp \left\{\frac{g^{\beta}(t)}{\theta}\right\} E_{i}\left(-\frac{g^{\beta}(t)}{\theta}\right)\right. \\
& +\sum_{i=0}^{n-2} a_{i}\left\{\sum_{m=1}^{n-i-1} \frac{(m-1) !}{(n-i-1) !}\left(-\frac{g^{\beta}(t)}{\theta}\right)^{n-i-m-1}\right. \\
& \left.-\frac{1}{(n-i-1) !}\left(-\frac{g^{\beta}(t)}{\theta}\right)^{n-i-1} \exp \left(\frac{g^{\beta}(t)}{\theta}\right) E_{i}\left(-\frac{g^{\beta}(t)}{\theta}\right)\right\} \\
& \left.+\sum_{i=n+1}^{2 n} a_{i}(i-n) !\left(\frac{\theta}{g^{\beta}(t)}\right)^{i-n+1} \sum_{r=0}^{i-n} \frac{1}{r !}\left(\frac{g^{\beta}(t)}{\theta}\right)^{r}\right]-\exp \left(-\frac{2 g^{\beta}(t)}{\theta}\right)
\end{aligned}
$$


where $a_{i}=(-1)^{i}\left(\begin{array}{c}2 n \\ i\end{array}\right)$ and $-E_{i}(-x)=\int_{x}^{\infty} \frac{e^{-u}}{u} d u$. Then the MSE of PTE of $R(t)$ based on UMVUE is

$$
\begin{aligned}
M S E\left(\tilde{R}_{P T-U}\right)= & R-\varphi_{7}-\varphi_{8}^{2} \\
& +R_{0}(t)\left\{H_{2 n+2}\left(\lambda C_{1}\right)-H_{2 n+2}\left(\lambda C_{2}\right)\right\}\left\{1-\left\{H_{2 n+2}\left(\lambda C_{1}\right)-H_{2 n+2}\left(\lambda C_{2}\right)\right\}\right\} \\
& +2 \varphi_{8} R(t)+2 R_{0}(t)\left\{H_{2 n+2}\left(\lambda C_{1}\right)-H_{2 n+2}\left(\lambda C_{2}\right)\right\}\left(\varphi_{8}-R(t)\right) \\
& +\left[R_{0}(t)\left\{H_{2 n+2}\left(\lambda C_{1}\right)-H_{2 n+2}\left(\lambda C_{2}\right)\right\}-\varphi_{8}\right]^{2}
\end{aligned}
$$

Now, we derive bias and MSE expressions of PTEs of $P$ based on MLE and UMVUE.

$$
\begin{aligned}
\operatorname{Bias}\left(\hat{P}_{P T-M L}\right) & =E(\hat{P})-E(\hat{P} I(B))+P_{0} E(I(B))-P \\
E(\hat{P}) & =E\left(\frac{\hat{\theta}_{1}}{\hat{\theta}_{1}+\hat{\theta}_{2}}\right)=E(\hat{Q})(\text { say })
\end{aligned}
$$

Following the approach by Constantine, Tse, and Karson (1986), we obtain the PDF of $\hat{Q}$ by transformation into two new independent RVs $r>0$ and $\theta \in\left(0, \frac{\pi}{2}\right)$ where $\hat{\theta}_{1}=\frac{\theta_{1} r \sin ^{2} \theta}{n+1}$ and $\hat{\theta}_{2}=\frac{\theta_{2} r \cos ^{2} \theta}{m+1}$. Here $r$ and $\theta$ are polar coordinates representing the distance from the origin and the angle between the line segment from the origin and the positive $x$-axis respectively.

Putting $\varphi=\cos ^{2} \theta$ and $\rho=\frac{\theta_{2}}{\theta_{1}}$, the PDF of $\hat{Q}=\left[1+\rho\left(\frac{n+1}{m+1}\right)\left(\frac{\varphi}{1-\varphi}\right)\right]^{-1}$ is

$$
\begin{aligned}
g(q)= & \frac{1}{B(n+1, m+1)}\left[\rho\left(\frac{n+1}{m+1}\right)\right]^{n+1} \\
& \times \frac{q^{n}(1-q)^{m}}{(1+\epsilon q)^{n+m+2}}, 0<q<1, \epsilon=\rho\left(\frac{n+1}{m+1}\right)-1
\end{aligned}
$$

where $B(a, b)$ is the Beta function with parameters $a$ and $b$.

When $\epsilon=0,(2.30)$ gives

$$
E\left(\hat{Q}^{l}\right)=\frac{B(n+l+1, m+1)}{B(n+1, m+1)}
$$

When $\epsilon \neq 0,(2.30)$ yields on substituting $1+\epsilon q=t$,

$$
\begin{aligned}
E\left(\hat{Q}^{l}\right)= & \frac{1}{B(n+1, m+1)}\left[\rho\left(\frac{n+1}{m+1}\right)\right]^{n+1} \frac{1}{\epsilon^{n+m+l+1}} \\
& \times \int_{1}^{\omega}(t-1)^{n+l}(\omega-t)^{m} t^{-(n+m+2)} d t
\end{aligned}
$$

where $\omega=1+\epsilon$. Two binomial expansions further simplify (2.32) to

$$
\begin{aligned}
E\left(\hat{Q}^{l}\right)= & \frac{(-1)^{n+1} \omega^{-l}\left(1-\omega^{-1}\right)^{-n-m-l-1}}{B(n+1, m+1)} \sum_{j=0}^{m}(-1)^{j}\left(\begin{array}{c}
m \\
j
\end{array}\right) \omega^{-j} \\
& \times \sum_{k=0}^{n+l}(-1)^{k}\left(\begin{array}{c}
n+l \\
k
\end{array}\right) I(w ; j+k-n-m-2)
\end{aligned}
$$

where

$$
I(\omega ; z)= \begin{cases}\frac{\omega^{z+1}-1}{z+1}, & z \neq-1 \\ \log (\omega), & z=-1\end{cases}
$$

Thus,

$$
E(\hat{P})= \begin{cases}\frac{n+1}{n+m+2}, & \omega=1 \\ \varphi_{9}, & \omega \neq 1\end{cases}
$$


where

$$
\begin{aligned}
\varphi_{9}= & \frac{(-1)^{n+1} \omega^{-1}\left(1-\omega^{-1}\right)^{-n-m-2}}{B(n+1, m+1)} \sum_{j=0}^{m}(-1)^{j}\left(\begin{array}{c}
m \\
j
\end{array}\right) \omega^{-j} \\
& \times \sum_{k=0}^{n+1}(-1)^{k}\left(\begin{array}{c}
n+1 \\
k
\end{array}\right) I(\omega ; j+k-n-m-2)
\end{aligned}
$$

Now,

$$
\begin{aligned}
E(\hat{P} I(B)) & =\frac{1}{B(n+1, m+1)}\left(\frac{n+1}{m+1}\right)^{n+1} \int_{C_{4}}^{C_{3}} \frac{u^{n}\left(1+\left(\frac{n+1}{m+1}\right) u\right)^{-n-m-2}}{\left(1+\frac{\theta_{2}}{\theta_{1} u}\right)} d u \\
& =\varphi_{10},(\text { say })
\end{aligned}
$$

then the bias of PTE of $P$ based on MLE is

$$
\operatorname{Bias}\left(\hat{P}_{P T-M L}\right)= \begin{cases}-\varphi_{10}+P_{0}\left\{F_{2(n+1), 2(m+1)}\left(C_{3}\right)-F_{2(n+1), 2(m+1)}\left(C_{4}\right)\right\}, & \omega=1 \\ \varphi_{9}-\varphi_{10}+P_{0}\left\{F_{2(n+1), 2(m+1)}\left(C_{3}\right)-F_{2(n+1), 2(m+1)}\left(C_{4}\right)\right\}-P, & \omega \neq 1\end{cases}
$$

and the MSE of PTE of $P$ based on MLE is

$$
\begin{aligned}
& \operatorname{MSE}\left(\hat{P}_{P T-M L}\right)= \\
& \qquad \begin{cases}\left(\frac{n+1}{n+m+2}\right)^{2}\left[\left(\frac{n+2}{n+1}\right)\left(\frac{n+m+2}{n+m+3}\right)-1\right]-\varphi_{11}+2 P\left(\varphi_{10}-P_{0} P(B)\right)+P_{0}^{2} P(B), & \omega=1 \\
\varphi_{12}-\varphi_{11}+2 P\left(\varphi_{10}-P_{0} P(B)\right)+P_{0}^{2} P(B)+P\left(P-2\left(\frac{n+1}{n+m+2}\right)\right), & \omega \neq 1\end{cases}
\end{aligned}
$$

where

$$
\begin{aligned}
P(B)= & \left\{F_{2(n+1), 2(m+1)}\left(C_{3}\right)-F_{2(n+1), 2(m+1)}\left(C_{4}\right)\right\} \\
\varphi_{11}= & \frac{1}{B(n+1, m+1)}\left(\frac{n+1}{m+1}\right)^{n+1} \int_{C_{4}}^{C_{3}} \frac{u^{n}\left(1+\left(\frac{n+1}{m+1}\right) u\right)^{-n-m-2}}{\left(1+\frac{\theta_{2}}{\theta_{1} u}\right)^{2}} d u \\
\varphi_{12}= & \frac{(-1)^{n} \omega^{-2}\left(1-\omega^{-1}\right)^{-n-m-3}}{B(n+1, m+1)} \sum_{j=0}^{m}(-1)^{j}\left(\begin{array}{c}
m \\
j
\end{array}\right) \omega^{-j} \\
& \times \sum_{k=0}^{n+2}(-1)^{k}\left(\begin{array}{c}
n+2 \\
k
\end{array}\right) I(\omega ; j+k-n-m-2)
\end{aligned}
$$

It is easy to see that the bias of PTE of $P$ based on UMVUE is

$$
\operatorname{Bias}\left(\tilde{P}_{P T-U}\right)= \begin{cases}P_{0} P(B)-\varphi_{13}, & v \leq 1 \\ P_{0} P(B)-\varphi_{14}, & v>1\end{cases}
$$

where $V=\frac{g^{\beta}\left(R_{n}\right)}{g^{\beta}\left(R_{n}^{*}\right)}$.

Denoting by $\varphi_{13}=\sum_{i=0}^{m-1} \frac{(-1)^{i} m ! n !}{(m-i-1) !(n+i+1) !}\left(\frac{\theta_{1}(n+1)}{\theta_{2}(m+1)}\right)^{i+1} \int_{C_{4}}^{C_{3}} u^{i+1} \phi_{1}(u) d u$, where $\phi_{1}(\cdot)$ is the PDF of $F$ - distribution with $(2(n+1), 2(m+1))$ degrees of freedom and $\varphi_{14}=\sum_{i=0}^{n} \frac{(-1)^{i} n ! m !}{(n-i) !(m+i) !}$ 
$\times\left(\frac{\theta_{2}(m+1)}{\theta_{1}(n+1)}\right)^{i} \int_{C_{4}}^{C_{3}} u^{i} \phi_{2}(u) d u$, where $\phi_{2}(\cdot)$ is the PDF of $F$ - distribution with $(2(m+1), 2(n+1))$ degrees of freedom. To obtain the MSE of PTE of $P$ based on UMVUE, consider

$$
\begin{aligned}
E\left(\tilde{P}^{2}\right)= & E\left(\sum_{i=0}^{m-1} \sum_{j=0}^{m-1} a_{i} a_{j} V^{i+j+2} \mid V \leq 1\right) P(V \leq 1) \\
& +E\left(\sum_{i=0}^{n} \sum_{j=0}^{n} b_{i} b_{j} V^{-(i+j)} \mid V>1\right) P(V>1)
\end{aligned}
$$

where, $a_{i}=\frac{(-1)^{i} m ! n !}{(m-i-1) !(n+i+1) !}, b_{i}=\frac{(-1)^{i} n ! m !}{(n-i) !(m+i) !}$.

An explicit expression of $\operatorname{Var}(\tilde{P})$ depends on the evaluation of $E\left(V^{l} \mid V \leq 1\right) P(V \leq 1)$ and $E\left(V^{-l} \mid V>1\right) P(V>1)$ for $l \geq 0$. To evaluate them we first obtain the PDF of $V$. We have, $V=\frac{g^{\beta}\left(R_{n}\right)}{g^{\beta}\left(R_{m}^{*}\right)}$ which implies, $\frac{(m+1) \theta_{2}}{(n+1) \theta_{1}} V=\rho \frac{(m+1)}{(n+1)} V \sim F_{2(n+1), 2(m+1)}$. Thus we obtain the PDF of $V$ as

$$
h(v)=\frac{\rho^{n-1}}{B(n+1, m+1)} v^{n}(1+\rho v)^{-n-m-2}, v>0
$$

For $l>0$,

$$
E\left(V^{l} \mid V \leq 1\right) P(V \leq 1)=\int_{0}^{1} \frac{\rho^{n-1}}{B(n+1, m+1)} v^{n+l}(1+\rho v)^{-n-m-2} d v
$$

Substituting $r=(1+\rho v)^{-1}$, the binomial expansion of the integrand yields,

$$
E\left(V^{l} \mid V \leq 1\right) P(V \leq 1)=\frac{\rho^{-l}}{B(n+1, m+1)} \sum_{k=0}^{n+l}(-1)^{k}\left(\begin{array}{c}
n+l \\
k
\end{array}\right) \int_{Q}^{1} r^{m-l+k} d r
$$

where

$$
\int_{Q}^{1} r^{m-l+k} d r= \begin{cases}\frac{1-Q^{m-l+k+1}}{m-l+k+1}, & k \neq l-m-1 \\ -\log (Q), & k=l-m-1\end{cases}
$$

and $Q=\frac{1}{1+\rho}$.

Similarly we can obtain

$$
E\left(V^{-l} \mid V>1\right) P(V>1)=\frac{\rho^{l}}{B(n+1, m+1)} \sum_{k=0}^{m+l}(-1)^{k}\left(\begin{array}{c}
m+l \\
k
\end{array}\right) \int_{1-Q}^{1} r^{n-l+k} d r
$$

where

$$
\int_{1-Q}^{1} r^{n-l+k} d r= \begin{cases}\frac{1-(1-Q)^{n-l+k+1}}{n-l+k+1}, & k \neq l-m-1 \\ -\log (1-Q), & k=l-m-1\end{cases}
$$

Thus, $\operatorname{Var}(\tilde{P})=\varphi_{17}-P^{2}$ and $\operatorname{Var}(\tilde{P} I(B))= \begin{cases}\varphi_{15}-\varphi_{13}^{2}, & v \leq 1 \\ \varphi_{16}-\varphi_{14}^{2}, & v>1\end{cases}$ 
where

$$
\begin{aligned}
\varphi_{15}= & \sum_{i=0}^{m-1} \sum_{j=0}^{m-1} a_{i} a_{j}\left(\frac{\theta_{1}(n+1)}{\theta_{2}(m+1)}\right)^{i+j+2} \int_{C_{4}}^{C_{3}} u^{i+j+2} \phi_{1}(u) d u \\
\varphi_{16}= & \sum_{i=0}^{n} \sum_{j=0}^{n} b_{i} b_{j}\left(\frac{\theta_{2}(m+1)}{\theta_{1}(n+1)}\right)^{i+j} \int_{C_{4}}^{C_{3}} u^{i+j} \phi_{2}(u) d u \\
\varphi_{17}= & \sum_{i=0}^{m-1} \sum_{j=0}^{m-1} \frac{a_{i} a_{j} \rho^{-(i+j+2)}}{B(n+1, m+1)} \sum_{k=0}^{n+i+j+2}(-1)^{k}\left(\begin{array}{c}
n+i+j+2 \\
k
\end{array}\right) \int_{Q}^{1} r^{m+k-i-j-2} d r \\
& +\sum_{i=0}^{n} \sum_{j=0}^{n} \frac{b_{i} b_{j} \rho^{i+j}}{B(n+1, m+1)} \sum_{k=0}^{m+i+j}(-1)^{k}\left(\begin{array}{c}
m+i+j \\
k
\end{array}\right) \int_{1-Q}^{1} r^{n+k-i-j} d r
\end{aligned}
$$

Thus, we obtain the MSE of PTE of $P$ based on UMVUE as

$$
M S E\left(\tilde{P}_{P T-U}\right)= \begin{cases}\varphi_{17}-P^{2}-\varphi_{15}+2 P\left(\varphi_{13}-P_{0} P(B)\right)+P_{0}^{2} P(B), & v \leq 1 \\ \varphi_{17}-P^{2}-\varphi_{16}+2 P\left(\varphi_{14}-P_{0} P(B)\right)+P_{0}^{2} P(B), & v>1\end{cases}
$$

Comparing the performance of the proposed PTEs analytically is a complicated task because of their formulations. Therefore several figures as well as some numerical results are presented to discuss their performance. The relative efficiency of PTE of some parameter $\tau$ denoted by $\hat{\tau}_{P T-\delta}$ over its regular estimator denoted by $\hat{\tau}_{\delta}$ is defined as follows;

$$
e\left(\hat{\tau}_{P T-\delta} \mid \hat{\tau}_{\delta}\right)=\frac{M S E\left(\hat{\tau}_{\delta}\right)}{M S E\left(\hat{\tau}_{P T-\delta}\right)}
$$

where $\delta \in\{U, M L, E B\}$.

\subsection{Proposed preliminary test confidence interval}

In this section we construct PTCI of the scale parameter $\theta$. Suppose for known value of the shape parameter $\beta$, we are interested in testing the hypothesis

$$
\begin{aligned}
& H_{0}: \theta=\theta_{0} \\
& H_{1}: \theta \neq \theta_{0}
\end{aligned}
$$

Since $g^{\beta}\left(R_{n}\right)$ follows gamma distribution with parameters $(n+1, \theta)$, it is easy to obtain the $100(1-\alpha) \%$ equal tail confidence interval (ETCI) of $\theta$ as

$$
I_{E T C I}=\left(\frac{2 g^{\beta}\left(R_{n}\right)}{\chi_{2(n+1)}^{2}\left(1-\frac{\alpha}{2}\right)}, \frac{2 g^{\beta}\left(R_{n}\right)}{\chi_{2(n+1)}^{2}\left(\frac{\alpha}{2}\right)}\right)
$$

From (2.2) and (2.3), for $p=1$, we obtain the same expression for the MLE and UMVUE of $\theta$ as $\hat{\theta}=\frac{g^{\beta}\left(R_{n}\right)}{n+1}$. Then we can re-write $I_{E T C I}$ as

$$
I_{E T C I}=\left(C_{5} \hat{\theta}, C_{6} \hat{\theta}\right)
$$

Accordingly, we can define the PTCI of $\theta$ as

$$
I_{P T C I}=\left(C_{5} \hat{\theta}_{P T}, C_{6} \hat{\theta}_{P T}\right)
$$

where $\hat{\theta}_{P T}$ is the PTE of $\theta$ as defined in (2.9) and (2.10). 
If we let $\lambda=\frac{\theta}{\theta_{0}}$ and $T=\frac{2 g^{\beta}\left(R_{n}\right)}{\theta}$, then the CP of PTCI of $\theta$ is defined as

$$
\begin{aligned}
P\left(\theta \in I_{P T C I}\right)= & P\left(\theta \in\left(C_{5} \theta_{0}, C_{6} \theta_{0}\right) \mid \chi_{2(n+1)}^{2}\left(\frac{\alpha}{2}\right)<\frac{2 g^{\beta}\left(R_{n}\right)}{\theta_{0}}<\chi_{2(n+1)}^{2}\left(1-\frac{\alpha}{2}\right)\right) \\
& +P\left(\theta \in\left(C_{5} \hat{\theta}_{U}, C_{6} \hat{\theta}_{U}\right) \mid \frac{2 g^{\beta}\left(R_{n}\right)}{\theta_{0}}<\chi_{2(n+1)}^{2}\left(\frac{\alpha}{2}\right)\right) \\
& +P\left(\theta \in\left(C_{5} \hat{\theta}_{U}, C_{6} \hat{\theta}_{U}\right) \mid \frac{2 g^{\beta}\left(R_{n}\right)}{\theta_{0}}>\chi_{2(n+1)}^{2}\left(1-\frac{\alpha}{2}\right)\right)
\end{aligned}
$$

$$
\begin{aligned}
= & P\left(\left(C_{5}<\lambda<C_{6}\right) \mid \frac{\chi_{2(n+1)}^{2}\left(\frac{\alpha}{2}\right)}{\lambda}<T<\frac{\chi_{2(n+1)}^{2}\left(1-\frac{\alpha}{2}\right)}{\lambda}\right) \\
& +P\left(\chi_{2(n+1)}^{2}\left(\frac{\alpha}{2}\right)<T<\chi_{2(n+1)}^{2}\left(1-\frac{\alpha}{2}\right), T<\frac{\chi_{2(n+1)}^{2}\left(\frac{\alpha}{2}\right)}{\lambda}\right) \\
& +P\left(\chi_{2(n+1)}^{2}\left(\frac{\alpha}{2}\right)<T<\chi_{2(n+1)}^{2}\left(1-\frac{\alpha}{2}\right), T>\frac{\chi_{2(n+1)}^{2}\left(1-\frac{\alpha}{2}\right)}{\lambda}\right) \\
& =P\left(\frac{\chi_{2(n+1)}^{2}\left(\frac{\alpha}{2}\right)}{\lambda}<T<\frac{\chi_{2(n+1)}^{2}\left(1-\frac{\alpha}{2}\right)}{\lambda}\right) I_{\left(C_{5}, C_{6}\right)}(\lambda) \\
& +P\left(\chi_{2(n+1)}^{2}\left(\frac{\alpha}{2}\right)<T<\min \left\{\chi_{2(n+1)}^{2}\left(1-\frac{\alpha}{2}\right), \frac{\chi_{2(n+1)}^{2}\left(\frac{\alpha}{2}\right)}{\lambda}\right\}\right) \\
& +P\left(\max \left\{\chi_{2(n+1)}^{2}\left(\frac{\alpha}{2}\right), \frac{\chi_{2(n+1)}^{2}\left(1-\frac{\alpha}{2}\right)}{\lambda}\right\}<T<\chi_{2(n+1)}^{2}\left(1-\frac{\alpha}{2}\right)\right)
\end{aligned}
$$

Denoting the first term of the above equation by $C$, we obtain the CP of PTCI of $\theta$ as

$$
P\left(\theta \in I_{P T C I}\right)=\left\{\begin{array}{l}
C+1-\alpha, \quad 0<\lambda \leq \frac{\chi_{2(n+1)}^{2}\left(\frac{\alpha}{2}\right)}{\chi_{2(n+1)}^{2}\left(1-\frac{\alpha}{2}\right)} \text { or } \lambda>\frac{\chi_{2(n+1)}^{2}\left(1-\frac{\alpha}{2}\right)}{\chi_{2(n+1)}^{2}\left(\frac{\alpha}{2}\right)} \\
C+P\left(\chi_{2(n+1)}^{2}\left(\frac{\alpha}{2}\right)<T<\frac{\chi_{2(n+1)}^{2}\left(\frac{\alpha}{2}\right)}{\lambda}\right), \frac{\chi_{2(n+1)}^{2}\left(\frac{\alpha}{2}\right)}{\chi_{2(n+1)}^{2}\left(1-\frac{\alpha}{2}\right)}<\lambda \leq 1 \\
C+P\left(\frac{\chi_{2(n+1)}^{2}\left(1-\frac{\alpha}{2}\right)}{\lambda}<T<\chi_{2(n+1)}^{2}\left(1-\frac{\alpha}{2}\right)\right), \quad 1<\lambda \leq \frac{\chi_{2(n+1)}^{2}\left(1-\frac{\alpha}{2}\right)}{\chi_{2(n+1)}^{2}\left(\frac{\alpha}{2}\right)}
\end{array}\right.
$$

In order to find the EL of PTCI of $\theta$, we first obtain the length of PTCI of $\theta$ which is given by the following RV

$$
L_{P T}= \begin{cases}\theta_{0}\left(C_{6}-C_{5}\right), & \chi_{2(n+1)}^{2}\left(\frac{\alpha}{2}\right)<\frac{2 g^{\beta}\left(R_{n}\right)}{\theta}<\chi_{2(n+1)}^{2}\left(1-\frac{\alpha}{2}\right) \\ \hat{\theta}_{U}\left(C_{6}-C_{5}\right), & \frac{2 g^{\beta}\left(R_{n}\right)}{\theta_{0}}<\chi_{2(n+1)}^{2}\left(\frac{\alpha}{2}\right) \text { or } \frac{2 g^{\beta}\left(R_{n}\right)}{\theta_{0}}>\chi_{2(n+1)}^{2}\left(1-\frac{\alpha}{2}\right)\end{cases}
$$


Then the EL of the PTCI is given by

$$
\begin{aligned}
E\left(L_{P T}\right)= & E\left(L_{P T} \mid \chi_{2(n+1)}^{2}\left(\frac{\alpha}{2}\right)<\frac{2 g^{\beta}\left(R_{n}\right)}{\theta_{0}}<\chi_{2(n+1)}^{2}\left(1-\frac{\alpha}{2}\right)\right) \\
& \times P\left(\chi_{2(n+1)}^{2}\left(\frac{\alpha}{2}\right)<\frac{2 g^{\beta}\left(R_{n}\right)}{\theta_{0}}<\chi_{2(n+1)}^{2}\left(1-\frac{\alpha}{2}\right)\right) \\
& +E\left(L_{P T} \mid \frac{2 g^{\beta}\left(R_{n}\right)}{\theta_{0}}<\chi_{2(n+1)}^{2}\left(\frac{\alpha}{2}\right) \text { or } \frac{2 g^{\beta}\left(R_{n}\right)}{\theta_{0}}>\chi_{2(n+1)}^{2}\left(1-\frac{\alpha}{2}\right)\right) \\
& \times P\left(\frac{2 g^{\beta}\left(R_{n}\right)}{\theta_{0}}<\chi_{2(n+1)}^{2}\left(\frac{\alpha}{2}\right) \text { or } \frac{2 g^{\beta}\left(R_{n}\right)}{\theta_{0}}>\chi_{2(n+1)}^{2}\left(1-\frac{\alpha}{2}\right)\right) \\
= & \theta_{0}\left(C_{6}-C_{5}\right)\left[H_{2 n+2}\left(\frac{\chi_{2(n+1)}^{2}\left(1-\frac{\alpha}{2}\right)}{\lambda}\right)-H_{2 n+2}\left(\frac{\chi_{2(n+1)}^{2}\left(\frac{\alpha}{2}\right)}{\lambda}\right)\right. \\
& \left.+\lambda\left\{H_{2 n+4}\left(\frac{\chi_{2(n+1)}^{2}\left(\frac{\alpha}{2}\right)}{\lambda}\right)+1-H_{2 n+4}\left(\frac{\chi_{2(n+1)}^{2}\left(1-\frac{\alpha}{2}\right)}{\lambda}\right)\right\}\right]
\end{aligned}
$$

\subsection{Numerical findings}

In this section we consider Weibull distribution by substituting $g(x)=x$ in (1.1) and taking $\beta=3$. Since the relative efficiencies $e\left(\hat{\theta}_{P T-U}^{p} \mid \hat{\theta}_{U}^{p}\right)$ and $e\left(\hat{\theta}_{P T-M L}^{p} \mid \hat{\theta}_{M L}^{p}\right)$ depend on the sample size $(n+1)$ and level of significance $\alpha$, Table 1 shows the relative efficiency of $\theta_{P T-U}^{p}$ over $\theta_{U}^{p}$ and the interval of $\lambda=\frac{\theta_{0}}{\theta}$ for which this efficiency is greater than 1. Similarly, Table 2 shows the relative efficiency of $\hat{\theta}_{P T-M L}^{p}$ over $\hat{\theta}_{M L}^{p}$ and the interval of $\lambda$ for which this efficiency is greater than 1. Unlike, $e\left(\hat{\theta}_{P T-U}^{p} \mid \hat{\theta}_{U}^{p}\right)$ and $e\left(\hat{\theta}_{P T-M L}^{p} \mid \hat{\theta}_{M L}^{p}\right)$, the relative efficiency $e\left(\hat{\theta}_{P T-E B}^{p} \mid \hat{\theta}_{E B}^{p}\right)$ does not have a closed form and thus we use Monte Carlo simulation technique that involves the following steps:

I. For given values of $\mu$ and $\nu$, generate one sample from Inverted-Gamma $(\mu, \nu)$ and denote it as $\theta^{*}$.

II. For a specified value of $n$, generate $m$ random samples from Gamma $\left(n+1, \theta^{*}\right)$ to obtain $Y_{j}, j=1,2, \ldots, m$.

III. Compute, $\hat{\theta}_{E B}^{p}(j)=\frac{\Gamma(n+\nu-p+1)}{\Gamma(n+\nu+1)}\left(Y_{j}+\mu\right)^{p}, j=1,2, \ldots, m$.

IV. For a specified value of $\theta_{0}$, if we wish to test the hypothesis $H_{0}: \theta=\theta_{0}$, then using the test statistic (2.8) we get

$$
\hat{\theta}_{P T-E B}^{p}(j)=\hat{\theta}_{E B}^{p}(j)-\left(\hat{\theta}_{E B}^{p}(j)-\theta_{0}^{p}\right) I(A), j=1,2, \ldots, m .
$$

V. Compute $M S E=\frac{1}{m} \sum_{j=1}^{m}\left(\delta(j)-\theta^{* p}\right)^{2}$, where $\delta(j) \in\left\{\hat{\theta}_{E B}^{p}(j), \hat{\theta}_{P T-E B}^{p}(j)\right\}, j=1,2, \ldots, m$

For $\mu=2, \nu=4$ and $m=1000$, Table 3 shows the relative efficiency of $\hat{\theta}_{P T-E B}^{p}$ over $\hat{\theta}_{E B}^{p}$ and the interval of $\lambda$ for which this efficiency is greater than 1. For a particular sample size and level of significance, Figures 1, 2 and 3 show the relative efficiency of $\hat{\theta}_{P T-U}^{p}$ over $\hat{\theta}_{U}^{p}$, $\hat{\theta}_{P T-M L}^{p}$ over $\hat{\theta}_{M L}^{p}$ and $\hat{\theta}_{P T-E B}^{p}$ over $\hat{\theta}_{E B}^{p}$ respectively, with respect to $\lambda=\theta / \theta_{0}$. From Tables 1, 2 and 3 and Figures 1, 2 and 3, we observe that irrespective of the sample sizes and level of significance, the PTEs of $\theta^{p}$ based on MLE, UMVUE and EBE are more efficient estimators compared to their usual estimators respectively whenever $\lambda$ is close to 1 or in other words, the true value of the parameter is close to its guess value $\theta_{0}$.

For a fixed sample size $n=5$, Figure 4 shows the relative efficiency of $\hat{R}(t)_{P T-M L}$ over $\hat{R}(t)$ and the relative efficiency of $\tilde{R}(t)_{P T-U}$ over $\tilde{R}(t)$ for different time points and level of significance 0.05 with respect to $\lambda=\frac{R_{0}(t)}{R(t)}$. From this figure, it is clear that PTEs of $R(t)$ 
based on MLE and UMVUE outperform the usual estimators of $R(t)$ whenever $R(t)$ is close to $R_{0}(t)$.

Now we study the efficiency of PTEs of P based on MLE and UMVUE over the usual estimators of $P$ based on MLE and UMVUE. Suppose for different values of $\theta_{1}$ and $\theta_{2}$ we want to test the hypothesis $H_{0}: P=P_{0}$ against $H_{1}: P \neq P_{0}$ for fixed sample sizes $n=5$ and $m=7$. Then in Figure 5, relative efficiencies of PTEs of $P$ based on MLE and UMVUE over its usual estimators based on MLE and UMVUE respectively have been demonstrated with respect to $\lambda=\frac{P_{0}}{P}$. From this figure, it is clear that PTEs of $P$ based on MLE and UMVUE are more efficient than the usual estimators of $P$ based on MLE and UMVUE respectively in a small neighbourhood around $\lambda=1$, i.e. when the true value $P$ is close to the hypothesized value $P_{0}$.

In Figure 6, we show the CP of PTCI of the parameter $\theta$ with respect to $\lambda=\frac{\theta_{0}}{\theta}$ for a fixed sample size and level of significance. From the figure and the derived expression of the CP of PTCI of $\theta$, we observe that as the value of $\lambda$ tends to 0 or $\infty$, the CP of PTCI of $\theta$ tends to $1-\alpha$ and for some interval of $\lambda$, the CP of PTCI of $\theta$ is greater than $1-\alpha$. This domination interval is larger for smaller values of sample size. Thus, we can conclude that the $\mathrm{CP}$ of PTCI of $\theta$ is greater than the CP of ETCI of $\theta$ for some values of $\lambda$ in a specific interval around $\lambda=1$.

In Figure 7, we compare the scaled EL of PTCI of $\theta$ with the ETCI of $\theta$ with respect to $\lambda=\frac{\theta_{0}}{\theta}$. We observe from this figure that there exists an interval of $\lambda$ for which the EL of PTCI of $\theta$ is lower than its ETCI. This interval of $\lambda$ for which EL of PTCI of $\theta$ is lower decreases with an increase in sample size. We also note that as $\lambda$ tends to 0 or $\infty$, the EL of PTCI of $\theta$ tend to be close to the EL of ETCI of $\theta$.

Table 1: The relative efficiency of $\hat{\theta}_{P T-U}^{p}$ over $\hat{\theta}_{U}^{p}$ and the interval of $\lambda=\frac{\theta_{0}}{\theta}$ for which this efficiency is greater than 1

\begin{tabular}{|c|c|c|c|}
\hline$\alpha$ & 0.01 & 0.05 & 0.1 \\
\hline$n$ & Efficiency(Interval) & Efficiency(Interval) & Efficiency(Interval) \\
\hline 7 & $9.7788[0.92316 .1516]$ & $3.1332[1.09664 .5794]$ & $2.0417\left[\begin{array}{lll}1.2029 & 3.9731\end{array}\right]$ \\
\hline 8 & $9.9641[0.75844 .4983]$ & $3.1742\left[\begin{array}{lll}0.8939 & 3.4238\end{array}\right]$ & $2.0629\left[\begin{array}{lll}0.9761 & 3.0010\end{array}\right]$ \\
\hline 9 & 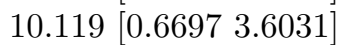 & $3.2083\left[\begin{array}{lll}0.7839 & 2.7928\end{array}\right]$ & $2.0807\left[\begin{array}{lll}0.8527 & 2.4685\end{array}\right]$ \\
\hline 10 & $10.25\left[\begin{array}{lll}0.6320 & 3.1292\end{array}\right]$ & $3.2371\left[\begin{array}{lll}0.7353 & 2.4626\end{array}\right]$ & $2.0957\left[\begin{array}{lll}0.7972 & 2.1920]\end{array}\right.$ \\
\hline 12 & $10.46\left[\begin{array}{lll}0.5809 & 2.5135\end{array}\right]$ & $3.2833\left[\begin{array}{lll}0.6691 & 2.0263\end{array}\right]$ & $2.1197\left[\begin{array}{lll}0.7214 & 1.8240]\end{array}\right.$ \\
\hline 15 & $10.687\left[\begin{array}{lll}0.5603 & 2.0853\end{array}\right]$ & $3.3332\left[\begin{array}{lll}0.6378 & 1.7254\end{array}\right]$ & $2.1458\left[\begin{array}{ll}0.6832 & 1.5723\end{array}\right]$ \\
\hline 20 & $10.936\left[\begin{array}{lll}0.3972 & 1.2441\end{array}\right]$ & $3.3875\left[\begin{array}{lll}0.4458 & 1.0593\end{array}\right]$ & $2.1742\left[\begin{array}{lll}0.4738 & 0.9786\end{array}\right]$ \\
\hline$\alpha$ & 0.15 & 0.2 & 0.25 \\
\hline$n$ & Efficiency(Interval) & Efficiency(Interval) & Efficiency(Interval) \\
\hline 7 & $1.6332\left[\begin{array}{lll}1.2799 & 3.6332\end{array}\right]$ & 1.4168 [1.3437 3.3969$]$ & $1.2835\left[\begin{array}{lll}1.4000 & 3.2157]\end{array}\right.$ \\
\hline 8 & $1.6476\left[\begin{array}{lll}1.0354 & 2.7614\end{array}\right]$ & $1.4277\left[\begin{array}{lll}1.0843 & 2.5937]\end{array}\right.$ & $1.2923\left[\begin{array}{lll}1.1274 & 2.4644\end{array}\right]$ \\
\hline 9 & $1.6597\left[\begin{array}{lll}0.9021 & 2.2831\end{array}\right]$ & $1.4368\left[\begin{array}{lll}0.9427 & 2.1527]\end{array}\right.$ & $1.2996\left[\begin{array}{lll}0.9784 & 2.0516\end{array}\right]$ \\
\hline 10 & $1.6699\left[\begin{array}{lll}0.8414 & 2.0362]\end{array}\right.$ & $1.4446\left[\begin{array}{lll}0.8777 & 1.9261]\end{array}\right.$ & $1.3058\left[\begin{array}{lll}0.9095 & 1.8404\end{array}\right]$ \\
\hline 12 & $1.6863\left[\begin{array}{lll}0.7585 & 1.7062\end{array}\right]$ & $1.457\left[\begin{array}{lll}0.7888 & 1.6222]\end{array}\right.$ & $1.3158\left[\begin{array}{lll}0.8152 & 1.5566\end{array}\right]$ \\
\hline 15 & $1.7041\left[\begin{array}{lll}0.7151 & 1.4820\end{array}\right]$ & $1.4705\left[\begin{array}{lll}0.7411 & 1.4171\end{array}\right]$ & $1.3267[0.76361 .3660]$ \\
\hline 20 & $1.7234\left[\begin{array}{lll}0.4934 & 0.9303\end{array}\right]$ & $1.4853\left[\begin{array}{ll}0.5092 & 0.8952\end{array}\right]$ & $1.3386\left[\begin{array}{lll}0.5228 & 0.8674\end{array}\right]$ \\
\hline
\end{tabular}

\subsection{An example on real data}

Now we consider a real data set which was also used in Lawless (1982). This data is concerning the data on time to breakdown of an insulating fluid between electrodes at a voltage of $34 \mathrm{kV}$ (minutes). The 19 times to breakdown are 
Table 2: The relative efficiency of $\hat{\theta}_{P T-M L}^{p}$ over $\hat{\theta}_{M L}^{p}$ and the interval of $\lambda=\frac{\theta_{0}}{\theta}$ for which this efficiency is greater than 1

\begin{tabular}{|c|c|c|c|}
\hline$\alpha$ & 0.01 & 0.05 & 0.1 \\
\hline$n$ & Efficiency(Interval) & Efficiency(Interval) & Efficiency(Interval) \\
\hline 7 & $10.15\left[\begin{array}{lll}0.8288 & 5.5230\end{array}\right]$ & $3.2341[0.98464 .1114]$ & $2.101\left[\begin{array}{lll}1.0800 & 3.5671\end{array}\right]$ \\
\hline 8 & $10.3\left[\begin{array}{l}0.7604 \\
4.5098]\end{array}\right.$ & $3.2659\left[\begin{array}{lll}0.8962 & 3.4326\end{array}\right]$ & $2.1169\left[\begin{array}{lll}0.9787 & 3.0087\end{array}\right]$ \\
\hline 9 & $10.426\left[\begin{array}{lll}0.6518 & 3.5068\end{array}\right]$ & $3.2923[0.76292 .7181]$ & $2.1302\left[\begin{array}{lll}0.8300 & 2.4025\end{array}\right]$ \\
\hline 10 & $10.533\left[\begin{array}{lll}0.6813 & 3.3733\end{array}\right]$ & $3.3146\left[\begin{array}{lll}0.7927 & 2.6547\end{array}\right]$ & $2.1414\left[\begin{array}{lll}0.8594 & 2.3630\end{array}\right]$ \\
\hline 12 & $\left.\begin{array}{lll}0.5683 & 2.4589\end{array}\right]$ & $3.3503\left[\begin{array}{lll}0.6546 & 1.9823\end{array}\right]$ & $2.1594\left[\begin{array}{ll}0.7057 & 1.7844\end{array}\right]$ \\
\hline 15 & $10.889\left[\begin{array}{lll}0.5412 & 2.0145]\end{array}\right.$ & $3.3889\left[\begin{array}{lll}0.6162 & 1.6668\end{array}\right]$ & $2.1788\left[\begin{array}{lll}0.6600 & 1.5189\end{array}\right]$ \\
\hline 20 & $11.093\left[\begin{array}{lll}0.4143 & 1.2977]\end{array}\right.$ & $3.431\left[\begin{array}{lll}0.4650 & 1.1050\end{array}\right]$ & $2.1999\left[\begin{array}{lll}0.4943 & 1.0208\end{array}\right]$ \\
\hline$\alpha$ & 0.15 & 0.2 & 0.25 \\
\hline$n$ & Efficiency(Interval) & Efficiency(Interval) & Efficiency(Interval) \\
\hline 7 & 1.677 [1.1491 3.2619] & $1.4522\left[\begin{array}{lll}1.2064 & 3.0498\end{array}\right]$ & $1.3134\left[\begin{array}{lll}1.2569 & 2.8871\end{array}\right]$ \\
\hline 8 & $1.6875\left[\begin{array}{lll}1.0380 & 2.7685\end{array}\right]$ & $1.46\left[\begin{array}{ll}1.0871 & 2.6004\end{array}\right]$ & $1.3196\left[\begin{array}{ll}1.1303 & 2.4708\end{array}\right]$ \\
\hline 9 & $1.6963\left[\begin{array}{lll}0.8780 & 2.2221\end{array}\right]$ & $1.4665[0.91752 .0951]$ & $1.3247\left[\begin{array}{ll}0.9522 & 1.9968\end{array}\right]$ \\
\hline 10 & 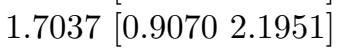 & $1.472\left[\begin{array}{lll}0.9462 & 2.0763]\end{array}\right]$ & $1.329\left[\begin{array}{lll}0.9804 & 1.9840\end{array}\right]$ \\
\hline 12 & $1.7157\left[\begin{array}{lll}0.7420 & 1.6691\end{array}\right]$ & $1.4808\left[\begin{array}{lll}0.7717 & 1.5870\end{array}\right]$ & $1.336\left[\begin{array}{lll}0.7975 & 1.5228\end{array}\right]$ \\
\hline 15 & $1.7286[0.6908$ 1.4317] & $1.4904\left[\begin{array}{lll}0.7159 & 1.3690\end{array}\right]$ & $1.3435[0.7377 \quad 1.3196]$ \\
\hline 20 & $1.7426\left[\begin{array}{lll}0.5146 & 0.9704\end{array}\right]$ & $1.5008\left[\begin{array}{lll}0.5311 & 0.9338\end{array}\right]$ & $1.3518\left[\begin{array}{lll}0.5453 & 0.9048\end{array}\right]$ \\
\hline
\end{tabular}

Table 3: The relative efficiency of $\hat{\theta}_{P T-E B}^{p}$ over $\hat{\theta}_{E B}^{p}$ and the interval of $\lambda=\frac{\theta_{0}}{\theta}$ for which this efficiency is greater than 1

\begin{tabular}{cccc}
\hline$\alpha$ & 0.01 & 0.05 & 0.1 \\
\hline$n$ & Efficiency(Interval) & Efficiency(Interval) & Efficiency(Interval) \\
\hline 7 & $8.1221[0.4643 \quad 3.0938]$ & $4.08[0.55152 .3031]$ & $2.8903[0.60501 .9982]$ \\
8 & $7.502[0.48222 .8599]$ & $3.9047[0.56832 .1768]$ & $2.7584[0.62061 .9079]$ \\
9 & $7.0921[0.49822 .6806]$ & $3.7608[0.58322 .0778]$ & $2.659[0.63441 .8365]$ \\
10 & $6.6345[0.51272 .5389]$ & $3.5683[0.59661 .9980]$ & $2.6337[0.64681 .7785]$ \\
12 & $5.9842[0.53752 .3257]$ & $3.2739[0.61911 .8749]$ & $2.4609[0.66751 .6877]$ \\
15 & $5.1106[0.56722 .1111]$ & $3.145[0.64571 .7467]$ & $2.3188[0.69161 .5917]$ \\
20 & $4.2836[0.60491 .8944]$ & $2.8152[0.67891 .6131]$ & $2.1749[0.72161 .4902]$ \\
\hline$\alpha$ & 0.15 & 0.2 & 0.25 \\
\hline$n$ & Efficiency(Interval) & Efficiency(Interval) & Efficiency(Interval) \\
\hline 7 & $2.5383[0.64371 .8272]$ & $2.2744[0.67581 .7084]$ & $2.0812[0.70411 .6173]$ \\
8 & $2.4285[0.65831 .7556]$ & $2.1807[0.68941 .6490]$ & $2.0004[0.71671 .5668]$ \\
9 & $2.355[0.67111 .6986]$ & $2.1497[0.70141 .6015]$ & $1.9416[0.72791 .5264]$ \\
10 & $2.2755[0.68271 .6521]$ & $2.0786[0.71211 .5627]$ & $1.8906[0.73791 .4932]$ \\
12 & $2.1534[0.70181 .5787]$ & $1.9599[0.72991 .5010]$ & $1.784[0.75431 .4403]$ \\
15 & $2.0116[0.72401 .5003]$ & $1.8595[0.75031 .4346]$ & $1.7273[0.77311 .3829]$ \\
20 & $1.873[0.75131 .4166]$ & $1.7361[0.77541 .3632]$ & $1.619[0.79611 .3209]$ \\
\hline
\end{tabular}




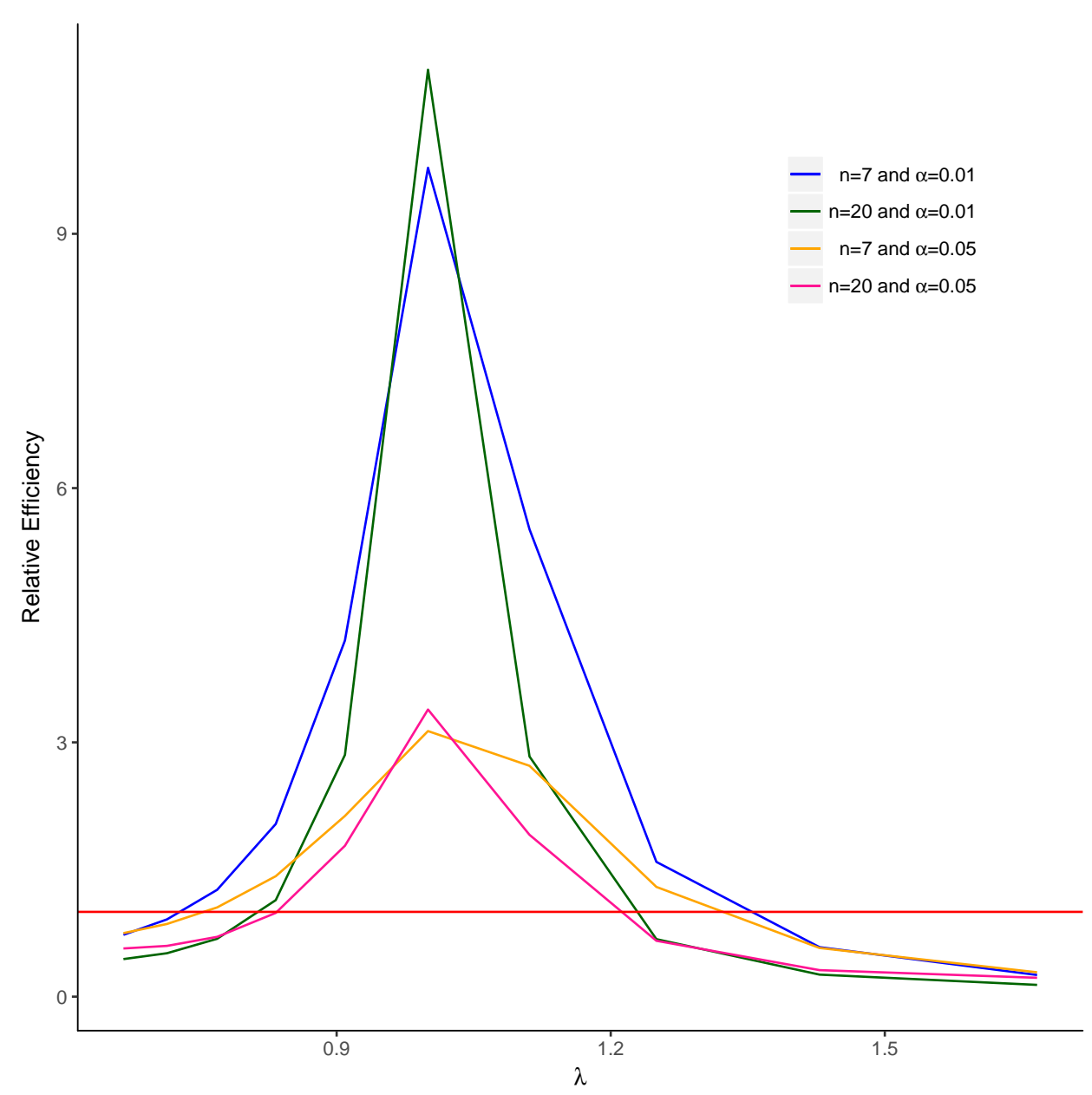

Figure 1. Relative efficiency of $\hat{\theta}_{P T-U}$ with respect to $\lambda=\frac{\theta_{0}}{\theta}$

Therefore, we observe the following 7 upper record values

\section{$\begin{array}{lllllll}0.96 & 4.15 & 8.01 & 31.75 & 33.91 & 36.71 & 72.89\end{array}$}

We first apply Kolmogorov-Smirnov (K-S) test and chi-square test to check whether for a fixed voltage level, time to breakdown has a Weibull distribution. Considering Weibull distribution as a lifetime model for the complete data, the computed K-S statistic is 0.1616 with a $p$-value of 0.6462 . The computed chi-square statistic is 0.5369 with a $p$-value of 0.4637 . Both the tests indicate that Weibull Distribution is suitable for the data at $5 \%$ level of significance.

Using the method of Profile log-likelihood before applying Newton-Raphson method, the maximum likelihood estimate of the parameter of Weibull distribution with shape parameter $\beta$ is $\hat{\beta}=0.7708$. Based on these upper record values we have $\hat{\theta}_{M L}=3.8962$. Consider the hypothesis

$$
\begin{aligned}
& H_{0}: \theta=3.5 \\
& H_{1}: \theta \neq 3.5
\end{aligned}
$$

The computed test-statistic is $\frac{2 g^{\hat{\beta}}\left(R_{n}\right)}{\theta_{0}}=15.5850$ which lies in the confidence interval $(5.6286$ 26.1189). Thus, we do not reject the null hypothesis at $5 \%$ level of significance which indicates that $\hat{\theta}_{P T-M L}=3.5$. The estimated value of $\lambda$ is $\hat{\lambda}=\frac{\theta_{0}}{\hat{\theta}_{M L}}=0.8983$, which falls in its range (0.5360 2.4872). We can also verify that the value of $\theta_{0}$ falls in the interval (2.3249 10.7880). 


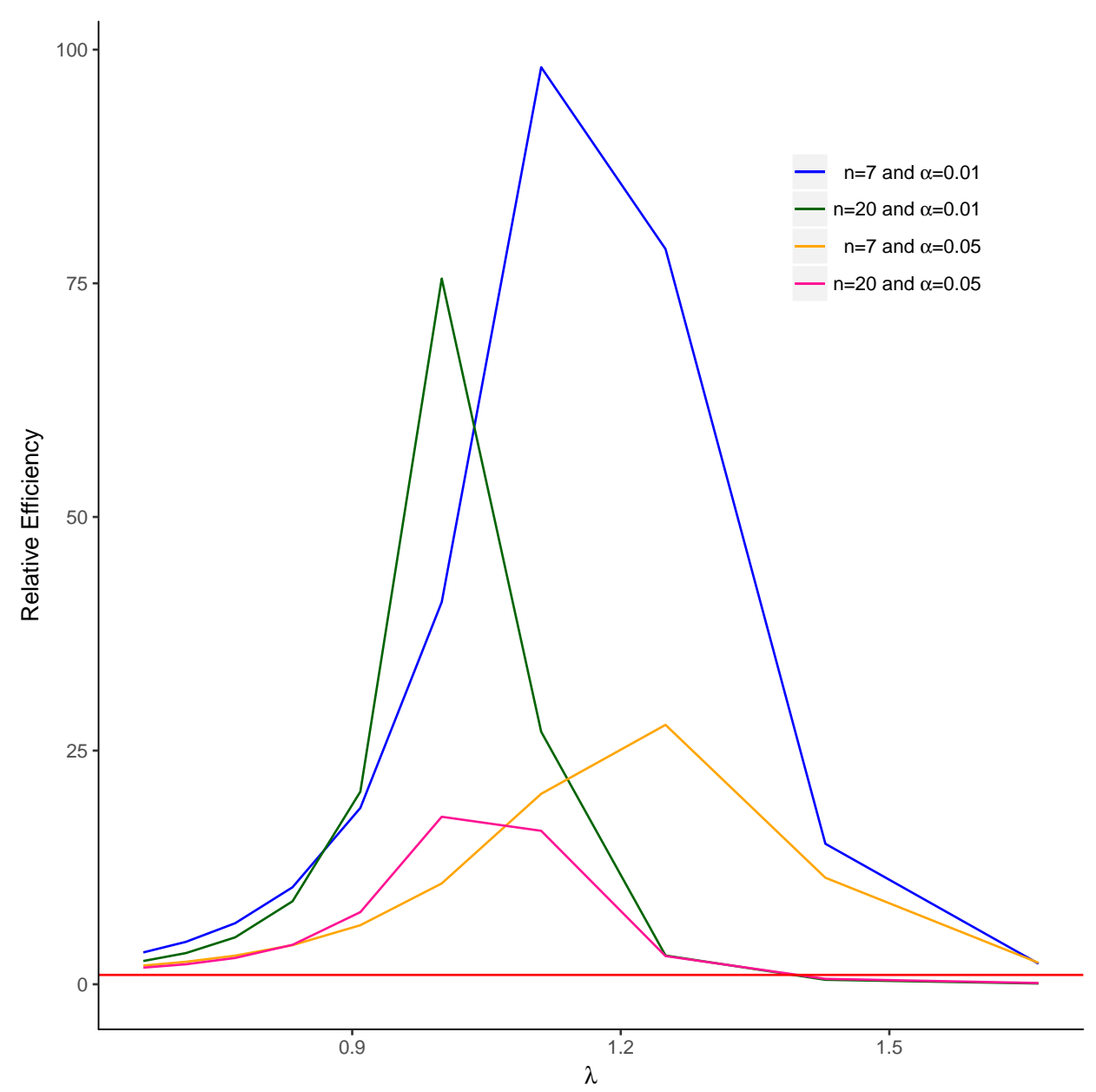

Figure 2. Relative efficiency of $\hat{\theta}_{P T-M L}$ with respect to $\lambda=\frac{\theta_{0}}{\theta}$

\subsection{Discussion on the proposed estimation methods}

In Section 2, we exhibited various PTEs for estimation of the powers of the parameter of interest and reliability functions of the Moore and Bilikam (1978) family of lifetime distribution. The exact bias and MSE expressions have been derived. Also, a real example has been analyzed to illustrate the performance of the classical PTE. It can be concluded that all of the proposed PTEs dominate their corresponding usual estimators such as UMVUE, MLE and EBE in the neighbourhood of null hypothesis. The PTEs of $R(t)$ perform better than their usual estimators and similarly, for $P$, the PTEs turn out to be better in the neighbourhood of the null hypothesis. Whenever the true value of the parameter is close to hypothesized value, the PTCI of the parameter have a greater CP and a smaller EL compared to their ETCI. Thus, we were able to establish improved estimators and confidence intervals of the parameters.

\section{Estimation of parametric functions when the scale parameter is known}

From (2.1), the log-likelihood function of parameter $\beta$ given $R_{0}, R_{1}, \ldots, R_{n}$ is

$$
k(\beta)=(n+1) \log \beta-(n+1) \log \theta-\frac{g^{\beta}\left(R_{n}\right)}{\theta}+\sum_{i=0}^{n} \log \left(g^{\prime}\left(R_{i}\right) g^{\beta-1}\left(R_{i}\right)\right)
$$

Now, for known value of $\theta$ we obtain the MLE of $\beta$ by employing Newton-Raphson iterative 


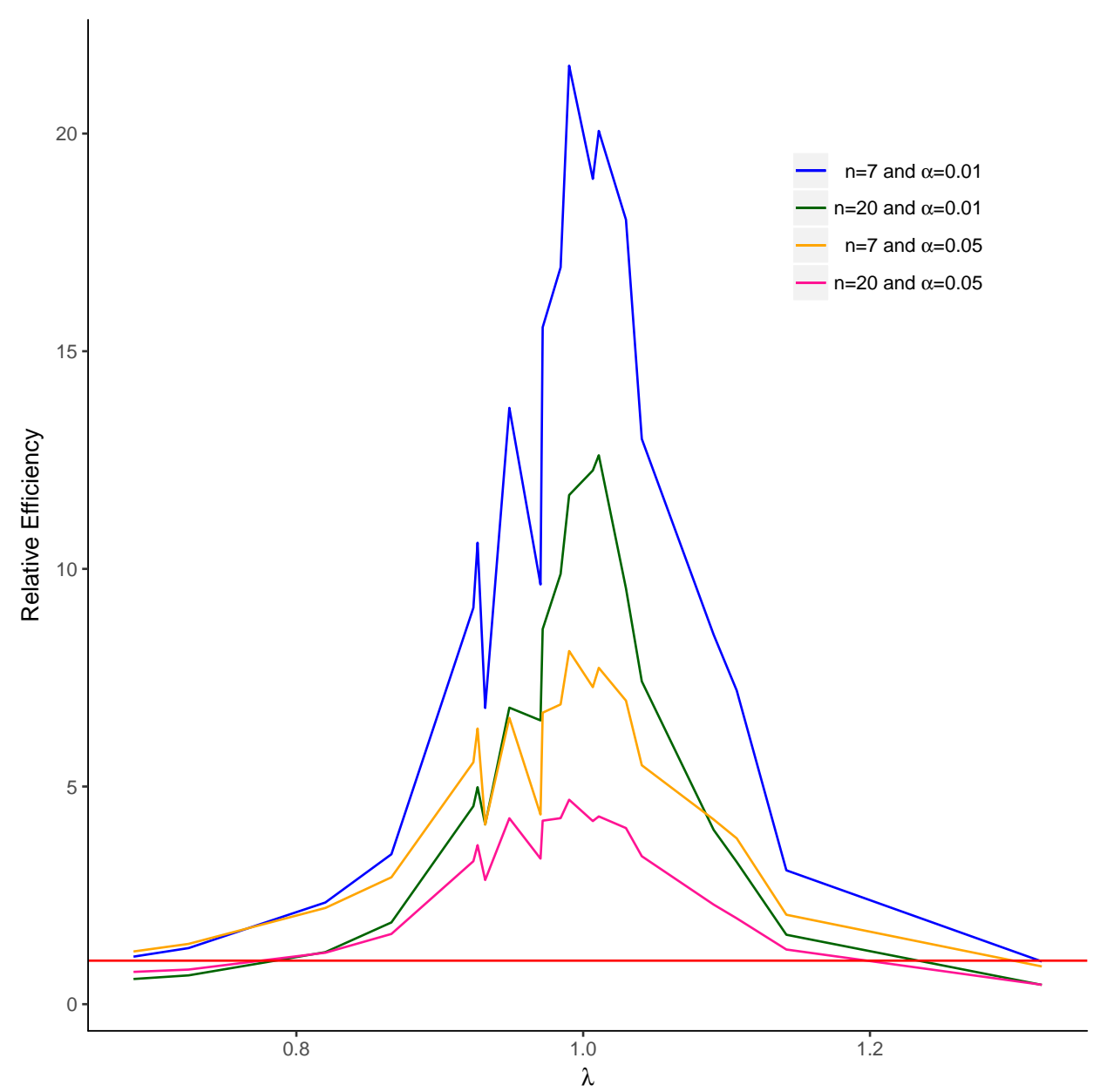

Figure 3. Relative efficiency of $\hat{\theta}_{P T-E B}$ with respect to $\lambda=\frac{\theta_{0}}{\theta}$

method which gives the $(l+1)$ th iterate of $\hat{\beta}$ as

$$
\hat{\beta}_{l+1}=\hat{\beta}_{l}-\frac{\frac{n+1}{\hat{\beta}_{l}}-\frac{g^{\hat{\beta}_{l}}\left(R_{n}\right)}{\theta} \log \left(g\left(R_{n}\right)\right)+\sum_{i=0}^{n} \log \left(g\left(R_{i}\right)\right)}{-\frac{n+1}{\hat{\beta}_{l}^{2}}-\frac{g^{\hat{\beta}_{l}}\left(R_{n}\right)}{\theta}\left\{\log \left(g\left(R_{n}\right)\right)\right\}^{2}}
$$

where $\hat{\beta}_{l}$ is the value of $\hat{\beta}$ at the $l$ th iteration.

Now, the Fisher information matrix is constructed as

$$
I(\beta)=E\left[-\frac{\partial^{2} k(\beta)}{\partial \beta^{2}}\right]
$$

Since it is a complicated task to obtain the above expected value, we use the observed Fisher information matrix by dropping the expectation sign, i.e.

$$
I(\hat{\beta})=\frac{n+1}{\hat{\beta}^{2}}+\frac{g^{\hat{\beta}}\left(R_{n}\right)}{\theta}\left\{\log \left(g\left(R_{n}\right)\right)\right\}^{2}
$$

where $\hat{\beta}$ is the MLE of $\beta$ obtained by the above Newton Raphson algorithm.

Since the analysis and construction of PTEs of the parametric functions are similar to that of the previous section, therefore, we skip this part and consider a more general case in the following section. 


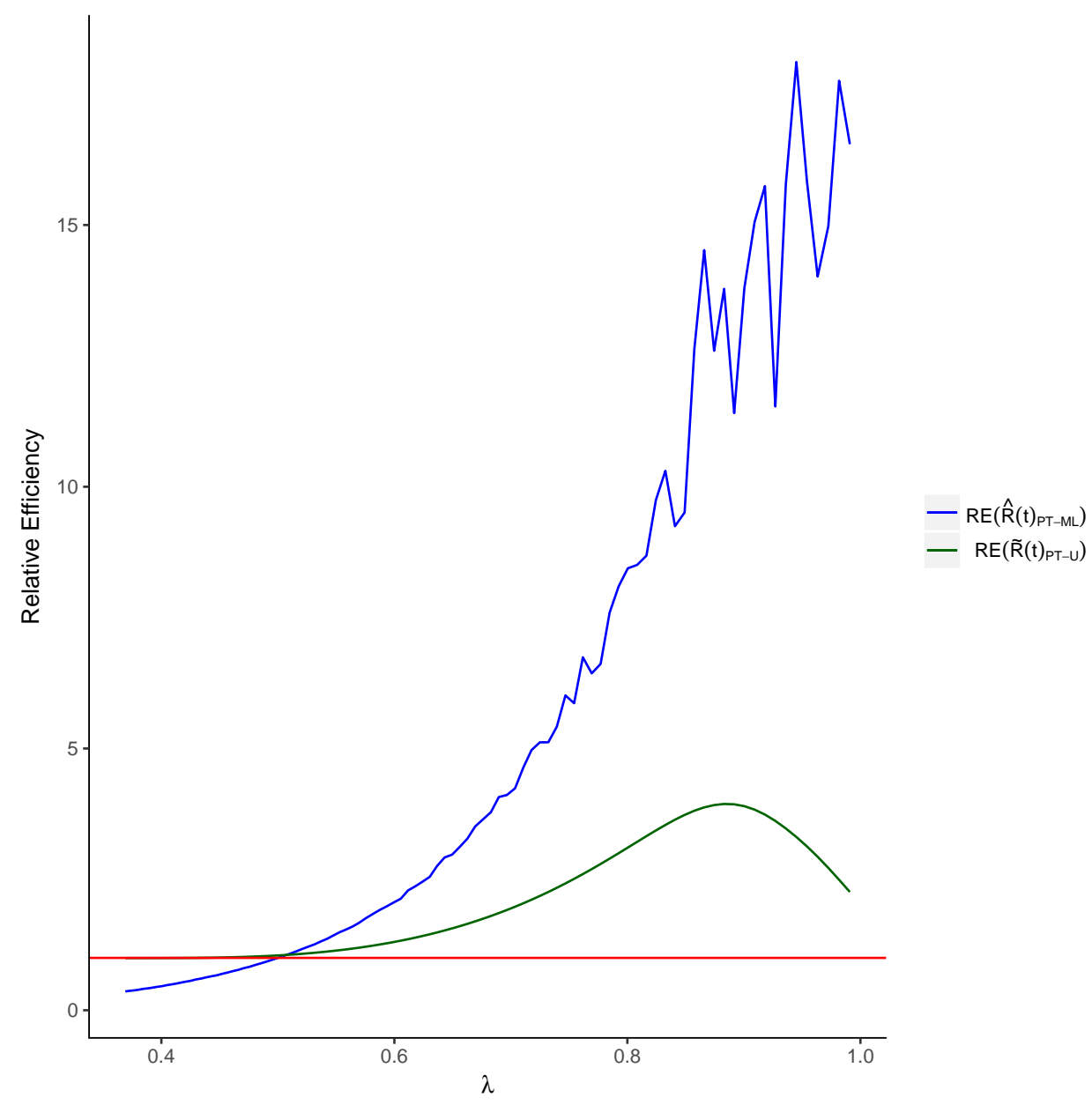

Figure 4. Relative efficiency of $\hat{R}(t)_{P T-M L}$ over $\hat{R}(t)$ and $\tilde{R}(t)_{P T-U}$ over $\tilde{R}(t)$ with respect to $\lambda=\frac{R_{0}(t)}{R(t)}$

\section{Estimation of parametric functions when both parameters are unknown}

In this section we consider the case when both the parameters $\theta$ and $\beta$ are unknown. Thus, the MLE of $\theta^{p}$ and $\beta$ are a solution of two nonlinear equations and we get

$$
\hat{\theta}^{p}=\left(\frac{g^{\hat{\beta}}\left(R_{n}\right)}{n+1}\right)^{p}
$$

where $\hat{\beta}$ is the MLE of $\beta$ that can be obtained by Newton-Raphson algorithm.

Now, we obtain the asymptotic bias and MSE of the estimators as it is difficult to obtain their exact expressions. In this regard, we compute the variance-covariance matrix of the MLE of $\theta$ and $\beta$ with the help of Fisher information matrix as

$$
I_{i j}=E\left[-\frac{\partial^{2} k(\theta, \beta)}{\partial \theta \partial \beta}\right], i, j=1,2
$$

where $k(\theta, \beta)$ is the same expression as $k(\beta)$ in Section 3, except that the parameter $\theta$ is now unknown. Since it is a complicated task to obtain the expected values, we approximate the variance-covariance matrix of the MLEs, which is obtained by simply dropping the expectation sign of the Fisher information matrix and inverting it. Thus,

$$
\left[\begin{array}{cc}
\operatorname{Var}(\hat{\theta}) & \operatorname{Cov}(\hat{\theta}, \hat{\beta}) \\
\operatorname{Cov}(\hat{\beta}, \hat{\theta}) & \operatorname{Var}(\hat{\beta})
\end{array}\right]=\left[\begin{array}{cc}
-\frac{\partial^{2} k(\theta, \beta)}{\partial^{2} \theta} & -\frac{\partial^{2} k(\theta, \beta)}{\partial \theta \partial \beta} \\
-\frac{\partial^{2} k(\theta, \beta)}{\partial \beta \partial \theta} & -\frac{\partial^{2} k(\theta, \beta)}{\partial^{2} \beta}
\end{array}\right]_{\hat{\theta}, \hat{\beta}}^{-1}
$$




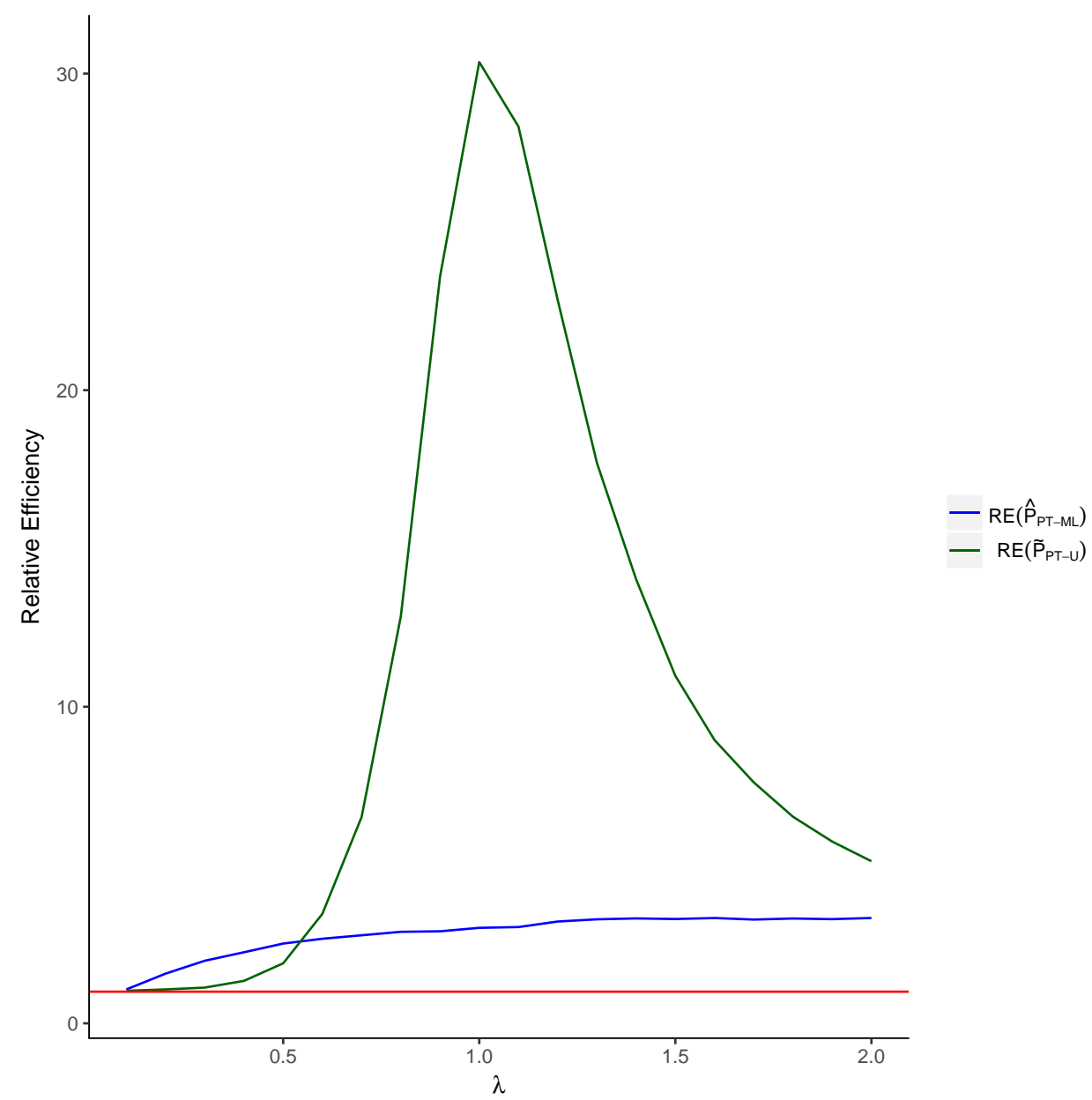

Figure 5. Relative efficiency of $\hat{R}_{P T-M L}$ over $\hat{P}$ and $\tilde{P}_{P T-U}$ over $\tilde{P}$ with respect to $\lambda=\frac{P_{0}}{P}$

where the elements are given by

$$
\begin{aligned}
& -\left.\frac{\partial^{2} k(\theta, \beta)}{\partial^{2} \theta}\right|_{\hat{\theta}, \hat{\beta}}=\frac{(n+1)^{3}}{\left\{g^{\hat{\beta}}\left(R_{n}\right)\right\}^{2}} \\
& -\left.\frac{\partial^{2} k(\theta, \beta)}{\partial \theta \partial \beta}\right|_{\hat{\theta}, \hat{\beta}}=-\left.\frac{\partial^{2} k(\theta, \beta)}{\partial \beta \partial \theta}\right|_{\hat{\theta}, \hat{\beta}}=\frac{-(n+1)^{2} \log \left(g\left(R_{n}\right)\right)}{g^{\hat{\beta}}\left(R_{n}\right)} \\
& -\left.\frac{\partial^{2} k(\theta, \beta)}{\partial^{2} \beta}\right|_{\hat{\theta}, \hat{\beta}}=\frac{n+1}{\hat{\beta}^{2}}+(n+1)\left\{\log \left(g\left(R_{n}\right)\right)\right\}^{2}
\end{aligned}
$$

\subsection{Proposed preliminary test estimator}

When both the parameters $\theta$ and $\beta$ are unknown, the exact distribution of the test statistics cannot be achieved and so we follow an approach by Gulati and Padgett (1994b,a, 1995). Suppose we have $m$ independent samples of upper record values of size $n+1$ as $R_{j 0}, R_{j 1}, \ldots, R_{j n}, j=1,2, \ldots m$. Then the MLE of the parameter $\theta^{p}$ is given by

$$
\hat{\theta}_{R}^{p}=\left(\frac{\sum_{j=1}^{m} g^{\hat{\beta}}\left(R_{j n}\right)}{m(n+1)}\right)^{p}
$$

Further suppose that there exists some prior information on the parameter in the form of $\theta=\theta_{0}$ and we are interested in estimating $\theta$ by incorporating such information. So we 


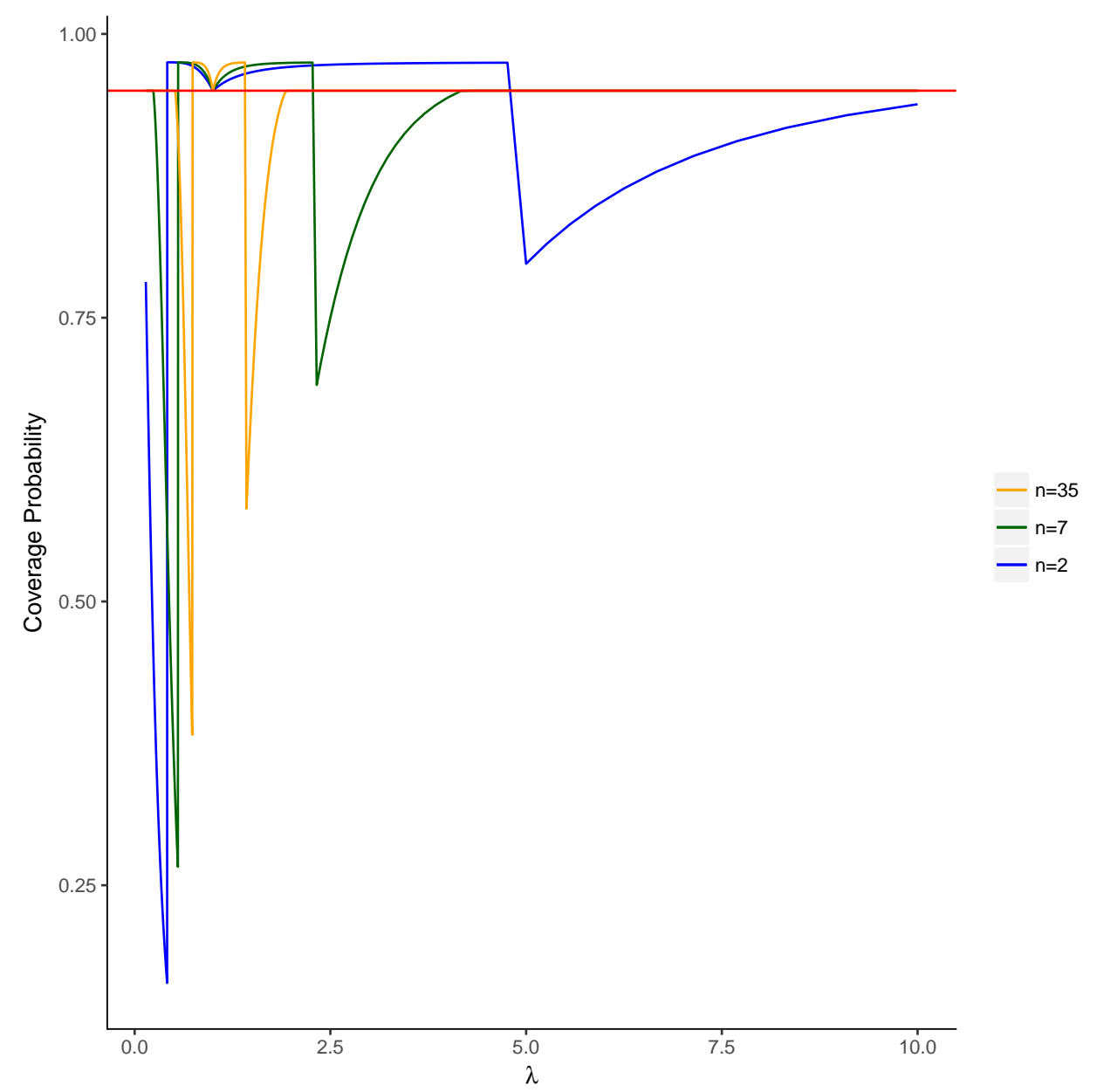

Figure 6. Coverage probability of PTCI of $\theta$ with respect to $\lambda=\frac{\theta_{0}}{\theta}$ for $\alpha=0.05$

consider the following simple hypothesis to check the validity of this information:

$$
\begin{aligned}
& H_{0}: \theta=\theta_{0} \\
& H_{1}: \theta \neq \theta_{0}
\end{aligned}
$$

Under $H_{0}, \frac{\sqrt{m}\left(\hat{\theta}_{R}-\theta_{0}\right)}{\sqrt{\operatorname{Var}\left(\hat{\theta}_{R}\right)}} \stackrel{\operatorname{asymp}}{\longrightarrow} N(0,1)$ and the test statistic is defined as

$$
L_{m-\theta}=\left(\frac{\sqrt{m}\left(\hat{\theta}_{R}-\theta_{0}\right)}{\sqrt{\operatorname{Var}\left(\hat{\theta}_{R}\right)}}\right)^{2}
$$

Under $H_{0}, L_{m-\theta}$ converges to central $\chi^{2}$ distribution with 1 degree of freedom as $m \rightarrow \infty$ while under the local alternative of the form

$$
H_{m}: \theta=\theta_{0}+\frac{\delta_{\theta}}{\sqrt{m}}
$$

$L_{m-\theta}$ converges to non-central $\chi^{2}$ distribution with non-centrality parameter

$$
\Delta_{\theta}^{2}=\left(\frac{\sqrt{m}\left(\theta-\theta_{0}\right)}{\sqrt{\operatorname{Var}\left(\hat{\theta}_{R}\right)}}\right)^{2}
$$

Based on the asymptotic distribution of $L_{m-\theta}$, the critical region is given by $L_{m-\theta}>\chi_{1}^{2}(\alpha)$ where $\alpha$ is the level of significance. Thus, we define PTE of $\theta^{p}$ as 


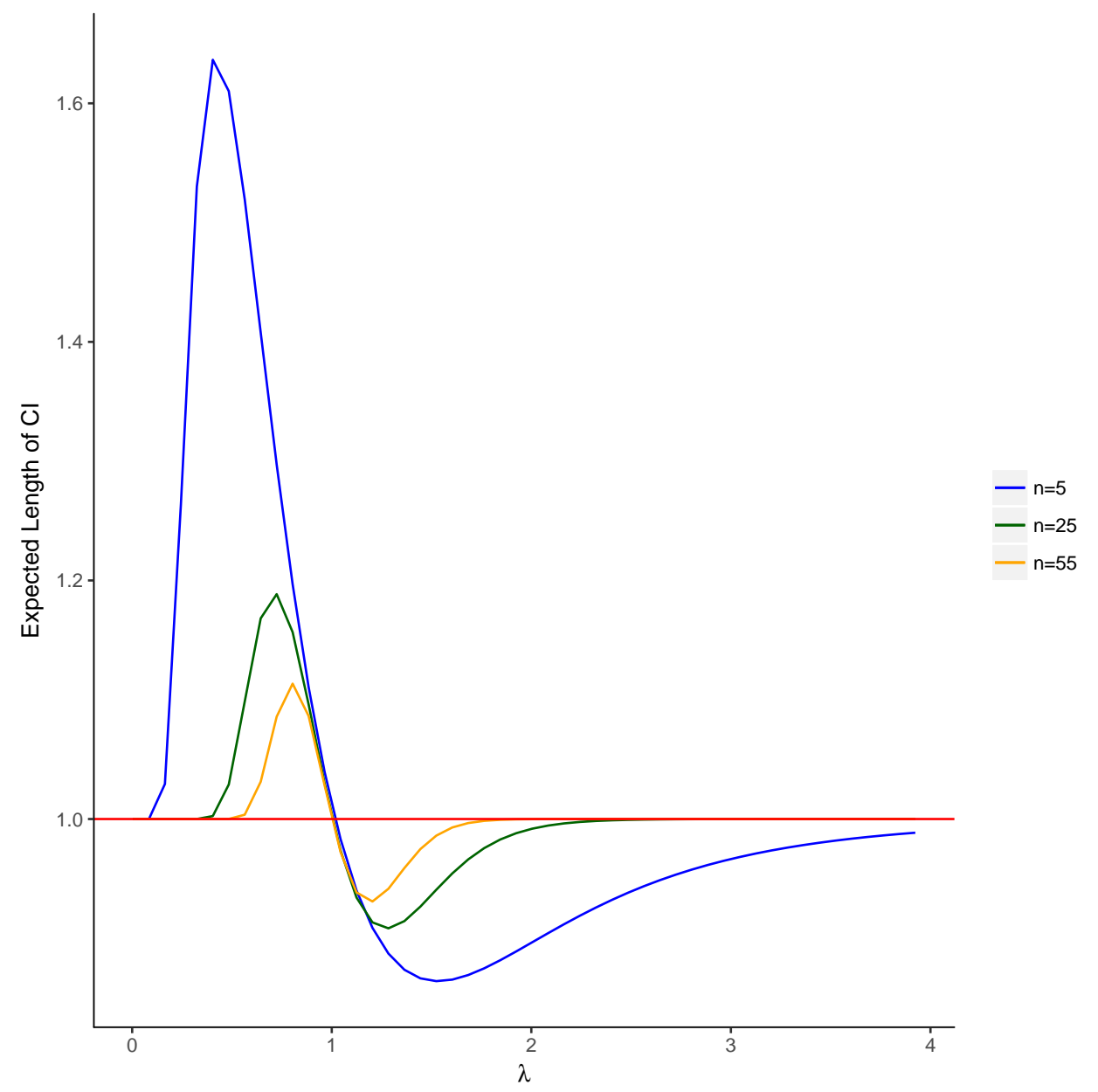

Figure 7. Comparison of expected length of ETCI with PTCI of $\theta$ with respect to $\lambda=\frac{\theta_{0}}{\theta}$ for $\alpha=0.05$

$$
\hat{\theta}_{P R-R}^{p}=\hat{\theta}_{R}^{p}-\left(\hat{\theta}_{R}^{p}-\theta_{0}^{p}\right) I\left(L_{m-\theta}<\chi_{1}^{2}(\alpha)\right)
$$

It is worthwhile to note that when the both parameters are unknown, we can construct the PTE based on Wald statistics. In this case the PTE can be defined as

$$
\hat{\eta}_{P T}=\hat{\eta}-\left(\hat{\eta}-\eta_{0}\right) I\left(W_{m}<w_{m, \alpha}\right)
$$

where $W_{m}=m\left(\hat{\eta}-\eta_{0}\right)^{T} I_{i j}^{-1}\left(\hat{\eta}-\eta_{0}\right), \hat{\eta}=\left(\begin{array}{c}\hat{\theta}_{R} \\ \hat{\beta}_{R}\end{array}\right), \eta_{0}=\left(\begin{array}{c}\theta_{0} \\ \beta_{0}\end{array}\right), w_{m, \alpha}$ is the $\alpha$ level critical value using the distribution of $W_{m}$, and $I_{i j}$ is the Fisher information matrix which can be approximated by the observed Fisher information matrix

$$
I\left(\hat{\theta}_{R}, \hat{\beta}_{R}\right)=\left[\begin{array}{ll}
-\frac{\partial^{2} k(\theta, \beta)}{\partial^{2} \theta} & -\frac{\partial^{2} k(\theta, \beta)}{\partial \theta \partial \beta} \\
-\frac{\partial^{2} k(\theta, \beta)}{\partial \beta \partial \theta} & -\frac{\partial^{2} k(\theta, \beta)}{\partial^{2} \beta}
\end{array}\right]_{\hat{\theta}_{R}, \hat{\beta}_{R}}
$$


and whose elements are given by

$$
\begin{aligned}
& -\left.\frac{\partial^{2} k(\theta, \beta)}{\partial^{2} \theta}\right|_{\hat{\theta}_{R}, \hat{\beta}_{R}}=\left(\frac{m(n+1)}{\sum_{j=1}^{m} g^{\hat{\beta}_{R}}\left(R_{j n}\right)}\right)^{2}\left[\frac{2 m(n+1) g^{\hat{\beta}_{R}}\left(R_{n}\right)}{\sum_{j=1}^{m} g^{\hat{\beta}}\left(R_{j n}\right)}-(n+1)\right] \\
& -\left.\frac{\partial^{2} k(\theta, \beta)}{\partial \theta \partial \beta}\right|_{\hat{\theta}_{R}, \hat{\beta}_{R}}=-\left.\frac{\partial^{2} k(\theta, \beta)}{\partial \beta \partial \theta}\right|_{\hat{\theta}_{R}, \hat{\beta}_{R}}=\frac{-(m(n+1))^{2} g^{\hat{\beta}_{R}} \log \left(g\left(R_{n}\right)\right)}{\left(\sum_{j=1}^{m} g^{\hat{\beta}_{R}}\left(R_{n}\right)\right)^{2}} \\
& -\left.\frac{\partial^{2} k(\theta, \beta)}{\partial^{2} \beta}\right|_{\hat{\theta}_{R}, \hat{\beta}_{R}}=\frac{n+1}{\hat{\beta}_{R}^{2}}+\frac{m(n+1) g^{\hat{\beta}_{R}}\left(R_{n}\right)\left\{\log \left(g\left(R_{n}\right)\right)\right\}^{2}}{\sum_{j=1}^{m} g^{\hat{\beta}_{R}}\left(R_{j n}\right)}
\end{aligned}
$$

Next, by invariance property of MLE, the MLE of the reliability function $R(t)$ under this approach is given by

$$
\hat{R}(t)_{R}=\exp \left(\frac{-g^{\hat{\beta}}(t)}{\hat{\theta}_{R}}\right)
$$

Further suppose that there exists some prior information on $R(t)$ in the form of $R(t)=R_{0}$ and we consider the following simple hypothesis to check the validity of this information:

$$
\begin{aligned}
& H_{0}: R(t)=R_{0} \\
& H_{1}: R(t) \neq R_{0}
\end{aligned}
$$

Under $H_{0}, \frac{\sqrt{m}\left(\hat{R}\left(t_{R}-R_{0}\right)\right.}{\sqrt{\operatorname{Var}\left(\hat{R}(t)_{R}\right)}} \stackrel{\text { asymp }}{\longrightarrow} N(0,1)$ and the test statistic is defined as

$$
L_{m-R(t)}=\left(\frac{\sqrt{m}\left(\hat{R}(t)_{R}-R_{0}\right)}{\sqrt{\operatorname{Var}\left(\hat{R}(t)_{R}\right)}}\right)^{2}
$$

Under $H_{0}, L_{m-R(t)}$ converges to central $\chi^{2}$ distribution with 1 degree of freedom as $m \rightarrow \infty$ while under the local alternative of the form

$$
H_{m}: R(t)=R_{0}+\frac{\delta_{R(t)}}{\sqrt{m}}
$$

$L_{m-R(t)}$ converges to non-central $\chi^{2}$ distribution with non-centrality parameter

$$
\Delta_{R(t)}^{2}=\left(\frac{\sqrt{m}\left(R(t)-R_{0}\right)}{\sqrt{\operatorname{Var}\left(\hat{R}(t)_{R}\right)}}\right)^{2}
$$

Based on the asymptotic distribution of $L_{m-R(t)}$, the critical region is given by $L_{m, R(t)}>\chi_{1}^{2}(\alpha)$ where $\alpha$ is the level of significance. Thus, we define PTE of $R(t)$ as

$$
\hat{R}(t)_{P T-R}=\hat{R}(t)_{R}-\left(\hat{R}(t)_{R}-R_{0}\right) I\left(L_{m-R(t)}<\chi_{1}^{2}(\alpha)\right)
$$

Finally, by invariance property of MLE, the MLE of the reliability function $P$ under this approach is given by

$$
\hat{P}_{R}=\frac{\hat{\theta}_{1 R}}{\hat{\theta}_{1 R}+\hat{\theta}_{2 R}}
$$

where $\hat{\theta}_{1 R}=\frac{\sum_{j=1}^{m} g^{\hat{\beta}}\left(R_{j n}\right)}{m(n+1)}$ and $\hat{\theta}_{2 R}=\frac{\sum_{j=1}^{m} g^{\hat{\beta}}\left(R_{j r}^{*}\right)}{m(r+1)}$ such that we have $m$ independent samples of $r+1$ upper record values $R_{j 0}^{*}, \ldots, R_{j r}^{*}, j=1,2, \ldots, m$ from the same family of distributions 
with $\beta_{1}=\beta_{2}=\beta$. Further suppose that there exists some prior information on $P$ in the form of $P=P_{0}$ and we consider the following simple hypothesis to check the validity of this information:

$$
\begin{aligned}
& H_{0}: P=P_{0} \\
& H_{1}: P \neq P_{0}
\end{aligned}
$$

Under $H_{0}, \frac{\sqrt{m}\left(\hat{P}_{R}-P_{0}\right)}{\sqrt{\operatorname{Var}\left(\hat{P}_{R}\right)}} \stackrel{a s y m p}{\longrightarrow} N(0,1)$ and the test statistic is defined as

$$
L_{m-P}=\left(\frac{\sqrt{m}\left(\hat{P}_{R}-P_{0}\right)}{\sqrt{\operatorname{Var}\left(\hat{P}_{R}\right)}}\right)^{2}
$$

Under $H_{0}, L_{m-P}$ converges to central $\chi^{2}$ distribution with 1 degree of freedom as $m \rightarrow \infty$ while under the local alternative of the form

$$
H_{m}: P=P_{0}+\frac{\delta_{P}}{\sqrt{m}}
$$

$L_{m-P}$ converges to non-central $\chi^{2}$ distribution with non-centrality parameter

$$
\Delta_{P}^{2}=\left(\frac{\sqrt{m}\left(P-P_{0}\right)}{\sqrt{\operatorname{Var}\left(\hat{P}_{R}\right)}}\right)^{2}
$$

Based on the asymptotic distribution of $L_{m-P}$, the critical region is given by $L_{m, P}>\chi_{1}^{2}(\alpha)$ where $\alpha$ is the level of significance. Thus, we define PTE of $P$ as

$$
\hat{P}_{P T-R}=\hat{P}_{R}-\left(\hat{P}_{R}-P_{0}\right) I\left(L_{m-P}<\chi_{1}^{2}(\alpha)\right)
$$

\subsection{Asymptotic bias and mean square error}

We derive the asymptotical distributional bias (ADB) and asymptotical distributional mean square error (ADMSE) of the proposed estimators in (4.1) and (4.4) under the local alternative given by (4.3). Following Saleh (2006) for any estimator $\hat{\theta}$ of $\theta$ we consider the following definitions of ADB (B) and ADMSE (M)

$$
\begin{aligned}
B(\hat{\theta}) & =\lim _{m \rightarrow \infty} E[\sqrt{m}(\hat{\theta}-\theta)] \\
M(\hat{\theta}) & =\lim _{m \rightarrow \infty} E\left[(\sqrt{m}(\hat{\theta}-\theta))^{2}\right]
\end{aligned}
$$

Lemma 4.1 (Saleh, 2006): If $Z \sim N(\Delta, 1)$ and $\varphi($.$) is a Borel measurable function, then$

1.E[Z. $\left.\varphi\left(Z^{2}\right)\right]=\Delta E\left[\varphi\left(\chi_{3}^{2}\left(\Delta^{2}\right)\right)\right]$

2. $E\left[Z^{2} \cdot \varphi\left(Z^{2}\right)\right]=E\left[\varphi\left(\chi_{3}^{2}\left(\Delta^{2}\right)\right)\right]+\Delta^{2} E\left[\varphi\left(\chi_{5}^{2}\left(\Delta^{2}\right)\right)\right]$

where $\chi_{d}^{2}\left(\Delta^{2}\right)$ is the non-central $\chi^{2} \mathrm{RV}$ with $d$ degrees of freedom and non centrality parameter $\Delta^{2}$.

From (4.14), the ADB of $\hat{\theta}_{R}$ and $\hat{\theta}_{P T-R}$ are obtained as follows:

$$
\begin{aligned}
B_{1}\left(\hat{\theta}_{R}\right) & =0 \\
B_{2}\left(\hat{\theta}_{P T-R}\right) & =\lim _{m \rightarrow \infty} E\left[\sqrt{m}\left(\hat{\theta}_{P T-R}-\theta\right)\right] \\
& =\lim _{m \rightarrow \infty} E\left[\sqrt{m}\left(\hat{\theta}_{R}-\left(\hat{\theta}_{R}-\theta_{0}\right) I\left(L_{m-\theta}<\chi_{1}^{2}(\alpha)\right)-\theta\right)\right] \\
& =\lim _{m \rightarrow \infty}\left[-E\left\{\sqrt{m}\left(\hat{\theta}_{R}-\theta_{0}\right) I\left(L_{m-\theta}<\chi_{1}^{2}(\alpha)\right)\right\}\right] \\
& \left.=-\sqrt{\operatorname{Var}\left(\hat{\theta}_{R}\right)} \lim _{m \rightarrow \infty} E\left[\frac{\sqrt{m}\left(\hat{\theta}_{R}-\theta_{0}\right)}{\sqrt{\operatorname{Var}\left(\hat{\theta}_{R}\right)}} I\left(\frac{\sqrt{m}\left(\hat{\theta}_{R}-\theta_{0}\right)}{\sqrt{\operatorname{Var}\left(\hat{\theta}_{R}\right)}}\right)^{2}<\chi_{1}^{2}(\alpha)\right)\right]
\end{aligned}
$$


Since $\frac{\sqrt{m}\left(\hat{\theta}_{R}-\theta_{0}\right)}{\sqrt{\operatorname{Var}\left(\hat{\theta}_{R}\right)}} \stackrel{\text { asymp }}{\longrightarrow} N\left(\Delta_{\theta}, 1\right)$, then by applying Lemma 4.1, taking $\varphi($.$) to be an indicator$ function, we have

$$
B_{2}\left(\hat{\theta}_{P T-R}\right)=-\delta_{\theta} H_{3}\left(\chi_{1}^{2}(\alpha), \Delta_{\theta}^{2}\right)
$$

where $H_{d}\left(., \Delta_{\theta}^{2}\right)$ is the CDF of non-central $\chi^{2}$ distribution with $d$ degrees of freedom and non-centrality parameter $\Delta_{\theta}^{2}$. Also, for ADMSE of $\hat{\theta}_{R}$ and $\hat{\theta}_{P T-R}$ we get

$$
M_{1}\left(\hat{\theta}_{R}\right)=\lim _{m \rightarrow \infty} E\left[\left(\sqrt{m}\left(\hat{\theta}_{R}-\theta\right)\right)^{2}\right]=\operatorname{Var}\left(\hat{\theta}_{R}\right)
$$

and

$$
\begin{aligned}
& M_{2}\left(\hat{\theta}_{P T-R}\right)=\lim _{m \rightarrow \infty} E\left[\left(\sqrt{m}\left(\hat{\theta}_{P T-R}-\theta\right)\right)^{2}\right] \\
& \left.\quad=\operatorname{Var}\left(\hat{\theta}_{R}\right)+\operatorname{Var}\left(\hat{\theta}_{R}\right) \lim _{m \rightarrow \infty} E\left[\left(\frac{\sqrt{m}\left(\hat{\theta}_{R}-\theta_{0}\right)}{\sqrt{\operatorname{Var}\left(\hat{\theta}_{R}\right)}}\right)^{2} I\left(\frac{\sqrt{m}\left(\hat{\theta}_{R}-\theta_{0}\right)}{\sqrt{\operatorname{Var}\left(\hat{\theta}_{R}\right)}}\right)^{2}<\chi_{1}^{2}(\alpha)\right)\right] \\
& \quad-2 \lim _{m \rightarrow \infty} E\left[\sqrt{m}\left(\hat{\theta}_{R}-\theta\right)\left\{\sqrt{m}\left(\left(\hat{\theta}_{R}-\theta_{0}\right) I\left(L_{m-\theta}<\chi_{1}^{2}(\alpha)\right)\right)\right\}\right] \\
& =\operatorname{Var}\left(\hat{\theta}_{R}\right)+\operatorname{Var}\left(\hat{\theta}_{R}\right) \lim _{m \rightarrow \infty} E\left[\left(\frac { \sqrt { m } ( \hat { \theta } _ { R } - \theta _ { 0 } ) } { \sqrt { \operatorname { V a r } ( \hat { \theta } _ { R } ) } ) ^ { 2 } I } \left(\frac{\sqrt{m}\left(\hat{\theta}_{R}-\theta_{0}\right)}{\left.\left.\left.\sqrt{\operatorname{Var}\left(\hat{\theta}_{R}\right)}\right)^{2}<\chi_{1}^{2}(\alpha)\right)\right]}\right.\right.\right. \\
& \left.+2 \operatorname{Var}\left(\hat{\theta}_{R}\right) \lim _{m \rightarrow \infty}\left(\frac{\sqrt{m}\left(\theta-\theta_{0}\right)}{\sqrt{\operatorname{Var}\left(\hat{\theta}_{R}\right)}}\right)\left[\frac{\sqrt{m}\left(\hat{\theta}_{R}-\theta\right)}{\sqrt{\operatorname{Var}\left(\hat{\theta}_{R}\right)}}\right) I\left(L_{m-\theta}<\chi_{1}^{2}(\alpha)\right)\right] \\
& -2 \operatorname{Var}\left(\hat{\theta}_{R}\right) \lim _{m \rightarrow \infty} E\left[\left(\frac{\sqrt{m}\left(\hat{\theta}_{R}-\theta\right)}{\sqrt{\operatorname{Var}\left(\hat{\theta}_{R}\right)}}\right)^{2} I\left(L_{m-\theta}<\chi_{1}^{2}(\alpha)\right)\right]
\end{aligned}
$$

From Lemma 4.1, we get

$$
M_{2}\left(\hat{\theta}_{P T-R}\right)=\operatorname{Var}\left(\hat{\theta}_{R}\right)\left[1-H_{3}\left(\chi_{1}^{2}(\alpha), \Delta_{\theta}^{2}\right)+\Delta_{\theta}^{2}\left\{2 H_{3}\left(\chi_{1}^{2}(\alpha), \Delta_{\theta}^{2}\right)-H_{5}\left(\chi_{1}^{2}(\alpha), \Delta_{\theta}^{2}\right)\right\}\right]
$$

The ADB and ADMSE of the proposed estimator in (4.5) can be obtained following Saleh (2006). Such PTEs may arise in situations where both the parameters are equally important for the experimenter. Since the theory of multivariate analysis using records needs more considerations, we leave the above idea for further research.

On similar lines we obtain from (4.14), the ADB of $\hat{R}(t)$ and $\hat{R}(t)_{P T-R}$ as follows:

$$
\begin{aligned}
B_{1}\left(\hat{R}(t)_{R}\right)=0 & \\
B_{2}\left(\hat{R}(t)_{P T-R}\right) & =\lim _{m \rightarrow \infty} E\left[\sqrt{m}\left(\hat{R}(t)_{P T-R}-R(t)\right)\right] \\
& =\lim _{m \rightarrow \infty} E\left[\sqrt{m}\left(\hat{R}(t)_{R}-\left(\hat{R}(t)_{R}-R_{0}\right) I\left(L_{m-R(t)}<\chi_{1}^{2}(\alpha)\right)-R(t)\right)\right] \\
& =-\delta_{R(t)} H_{3}\left(\chi_{1}^{2}(\alpha), \Delta_{R(t)}^{2}\right)
\end{aligned}
$$

Also, for ADMSE of $\hat{R}(t)_{R}$ and $\hat{R}(t)_{P T-R}$ we get

$$
M_{1}\left(\hat{R}(t)_{R}\right)=\lim _{m \rightarrow \infty} E\left[\left(\sqrt{m}\left(\hat{R}(t)_{R}-R(t)\right)\right)^{2}\right]=\operatorname{Var}\left(\hat{R}(t)_{R}\right)
$$

and from Lemma (4.1)

$$
\begin{aligned}
M_{2}\left(\hat{R}(t)_{P T-R}\right)= & \lim _{m \rightarrow \infty} E\left[\left(\sqrt{m}\left(\hat{R}(t)_{P T-R}-R(t)\right)\right)^{2}\right] \\
= & \operatorname{Var}\left(\hat{R}(t)_{R}\right)\left[1-H_{3}\left(\chi_{1}^{2}(\alpha), \Delta_{R(t)}^{2}\right)+\Delta_{R(t)}^{2}\left\{2 H_{3}\left(\chi_{1}^{2}(\alpha), \Delta_{R(t)}^{2}\right)\right.\right. \\
& \left.\left.-H_{5}\left(\chi_{1}^{2}(\alpha), \Delta_{R(t)}^{2}\right)\right\}\right]
\end{aligned}
$$


Finally, the ADB of $\hat{P}_{R}$ and $\hat{P}_{P T-R}$ are obtained as follows:

$$
\begin{aligned}
B_{1}\left(\hat{P}_{R}\right)=0 & \\
B_{2}\left(\hat{P}_{P T-R}\right) & =\lim _{m \rightarrow \infty} E\left[\sqrt{m}\left(\hat{P}_{P T-R}-P\right)\right] \\
& =\lim _{m \rightarrow \infty} E\left[\sqrt{m}\left(\hat{P}_{R}-\left(\hat{P}_{R}-P_{0}\right) I\left(L_{m-P}<\chi_{1}^{2}(\alpha)\right)-P\right)\right] \\
& =-\delta_{P} H_{3}\left(\chi_{1}^{2}(\alpha), \Delta_{P}^{2}\right)
\end{aligned}
$$

Also, for ADMSE of $\hat{P}_{R}$ and $\hat{P}_{P T-R}$ we get

$$
M_{1}\left(\hat{P}_{R}\right)=\lim _{m \rightarrow \infty} E\left[\left(\sqrt{m}\left(\hat{P}_{R}-P\right)\right)^{2}\right]=\operatorname{Var}\left(\hat{P}_{R}\right)
$$

and from lemma (4.1)

$$
\begin{aligned}
M_{2}\left(\hat{P}_{P T-R}\right) & =\lim _{m \rightarrow \infty} E\left[\left(\sqrt{m}\left(\hat{P}_{P T-R}-P\right)\right)^{2}\right] \\
& =\operatorname{Var}\left(\hat{P}_{R}\right)\left[1-H_{3}\left(\chi_{1}^{2}(\alpha), \Delta_{P}^{2}\right)+\Delta_{P}^{2}\left\{2 H_{3}\left(\chi_{1}^{2}(\alpha), \Delta_{P}^{2}\right)-H_{5}\left(\chi_{1}^{2}(\alpha), \Delta_{P}^{2}\right)\right\}\right]
\end{aligned}
$$

\subsection{Comparison}

In this section we analyze the ADMSE of the proposed estimators to study their relative performance. From (4.4) we see that if $L_{m-\theta} \rightarrow 0$ then $\hat{\theta}_{P T-R} \rightarrow \theta_{0}$ while $\hat{\theta}_{P T-R} \rightarrow \hat{\theta}_{R}$ as $L_{m-\theta} \rightarrow \infty$. The asymptotic relative efficiency (ARE) of $\hat{\theta}_{P T-R}$ over $\hat{\theta}_{R}$ is

$$
\begin{aligned}
\operatorname{ARE}\left(\hat{\theta}_{P T-R} \mid \hat{\theta}_{R}\right)= & \frac{M_{1}\left(\hat{\theta}_{R}\right)}{M_{2}\left(\hat{\theta}_{P T-R}\right)} \\
= & {\left[1-H_{3}\left(\chi_{1}^{2}(\alpha), \Delta_{\theta}^{2}\right)+\Delta_{\theta}^{2}\left\{2 H_{3}\left(\chi_{1}^{2}(\alpha), \Delta_{\theta}^{2}\right)\right.\right.} \\
& \left.\left.-H_{5}\left(\chi_{1}^{2}(\alpha), \Delta_{P}^{2}\right)\right\}\right]^{-1}
\end{aligned}
$$

Thus we can conclude that $\hat{\theta}_{P T-R}$ is a better estimator of $\theta$ than $\hat{\theta}_{R}$ whenever

$$
0 \leq \Delta_{\theta}^{2} \leq \frac{H_{3}\left(\chi_{1}^{2}(\alpha), \Delta_{\theta}^{2}\right)}{2 H_{3}\left(\chi_{1}^{2}(\alpha), \Delta_{\theta}^{2}\right)-H_{5}\left(\chi_{1}^{2}(\alpha), \Delta_{\theta}^{2}\right)}
$$

By the asymptotic normality of MLE for the parameter $\beta$, one may obtain similar results.

On similar lines, the ARE of $\hat{R}(t)_{P T-R}$ over $\hat{R}(t)_{R}$ is

$$
\begin{aligned}
A R E\left(\hat{R}(t)_{P T-R} \mid \hat{R}(t)_{R}\right)= & {\left[1-H_{3}\left(\chi_{1}^{2}(\alpha), \Delta_{R(t)}^{2}\right)+\Delta_{R(t)}^{2}\left\{2 H_{3}\left(\chi_{1}^{2}(\alpha), \Delta_{R(t)}^{2}\right)\right.\right.} \\
& \left.\left.-H_{5}\left(\chi_{1}^{2}(\alpha), \Delta_{R(t)}^{2}\right)\right\}\right]^{-1}
\end{aligned}
$$

and we can conclude that $\hat{R}(t)_{P T-R}$ is a better estimator of $R(t)$ than $\hat{R}(t)_{R}$ whenever

$$
0 \leq \Delta_{R(t)}^{2} \leq \frac{H_{3}\left(\chi_{1}^{2}(\alpha), \Delta_{R(t)}^{2}\right)}{2 H_{3}\left(\chi_{1}^{2}(\alpha), \Delta_{R(t)}^{2}\right)-H_{5}\left(\chi_{1}^{2}(\alpha), \Delta_{R(t)}^{2}\right)}
$$

Similarly, $\hat{P}_{P T-R}$ is a better estimator of $P$ than $\hat{P}_{R}$ whenever

$$
0 \leq \Delta_{P}^{2} \leq \frac{H_{3}\left(\chi_{1}^{2}(\alpha), \Delta_{P}^{2}\right)}{2 H_{3}\left(\chi_{1}^{2}(\alpha), \Delta_{P}^{2}\right)-H_{5}\left(\chi_{1}^{2}(\alpha), \Delta_{P}^{2}\right)}
$$




\section{Conclusion}

In this paper we proposed improved PTEs for estimation of the powers of the parameter of interest and the reliability functions of the Moore and Bilikam (1978) family of lifetime distribution. These estimators were derived for different cases when certain parameters are known and unknown. In order to check the relative efficiency of these new estimators, the exact bias and MSE expressions were derived. The conclusion of our extensive study is that all the proposed PTEs are superior to their corresponding usual estimators such as UMVUE, MLE and EBE whenever the true value of the parametric function is close to its prior guess value. In this study we also constructed PTCI for the parameter of the model under the assumption that shape parameter is known. We can easily conclude that the PTCI of the parameter have a greater coverage probability and a smaller expected length compared to their ETCI in the neighbourhood of the null hypothesis. Thus, we were able to establish improved estimators and confidence intervals of the parameters whenever the experimenter has some prior knowledge of the model parameters.

PTEs were also derived for a more general case when all the parameters were unknown. Studying the ARE of these PTEs with their corresponding usual estimators, we were able to establish the interval in which the proposed PTEs proved to be more efficient.

Interested researchers are recommended to develop asymptotic distributional bias and asymptotic distributional mean square error of PTE based on Wald statistics in the case when both the parameters are unknown. Such estimation procedures are more advantageous in situations where both the parameters are equally important for the experimenter. Since the theory of multivariate analysis needs more considerations, we leave the above idea for future research.

\section{Acknowledgements}

We are grateful to the associate editor and the reviewers for their fruitful suggestions and comments which lead to considerable improvement in this research paper.

\section{References}

Ahsanullah M (1980). "Linear Prediction of Record Values for the Two Parameter Exponential Distribution." Annals of the Institute of Statistical Mathematics, 32, 363-368.

Arashi M, Emadi M (2008). "Evidential Inference Based on Record Data and Inter-record Times." Statistical Papers, 49, 291-301.

Arnold BC, Balakrishnan N, Nagaraja HN (1992). A First Course In Order Statistics, volume 54. John Wiley \& Sons.

Balakrishnan N, Ahsanullah M, Chan PS (1995). "On the Logistic Record Values and Associated Inference." Journal of Applied Statistical Science, 2, 233-248.

Bancroft TA (1944). "On Biases in Estimation due to the Use of Preliminary Tests of Significance." The Annals of Mathematical Statistics, 15, 190-204.

Belaghi RA, Arashi M, Tabatabaey SMM (2014). "Improved Confidence Intervals for the Scale Parameter of Burr XII Model Based on Record Values." Computational Statistics, 29, 1153-1173.

Belaghi RA, Arashi M, Tabatabaey SMM (2015). "On the Construction of Preliminary Test Estimator Based on Record Values for the Burr XII Model." Communications in StatisticsTheory and Methods, 44, 1-23. 
Burr IW (1942). "Cumulative Frequency Functions." The Annals of Mathematical Statistics, 13, 215-232.

Burr IW, Cislak PJ (1968). "On a General System of Distributions I. Its Curve-shape Characteristics II. The Sample Median." Journal of the American Statistical Association, 63, 627-635.

Chandler KN (1952). "The Distribution and Frequency of Record Values." Journal of the Royal Statistical Society: Series B (Methodological), 14, 220-228.

Chaturvedi A, Malhotra A (2017a). "Estimation and Testing Procedures for the Reliability Functions of a Family of Lifetime Distributions Based on Records." International Journal of System Assurance Engineering and Management, 8, 836-848.

Chaturvedi A, Malhotra A (2017b). "Inference on the Parameters and Reliability Characteristics of Three Parameter Burr Distribution Based on Records." Applied Mathematics and Information Science, 11, 837-849.

Chen Z (2000). "A New Two-parameter Lifetime Distribution with Bathtub Shape or Increasing Failure Rate Function." Statistics \& Probability Letters, 49, 155-161.

Constantine K, Tse SK, Karson M (1986). "Estimation of P $(\mathrm{Y}<\mathrm{X})$ in the Gamma Case." Communications in Statistics-Simulation and Computation, 15, 365-388.

Glick N (1978). "Breaking Records and Breaking Boards." The American Mathematical Monthly, 85, 2-26.

Gulati S, Padgett WJ (1994a). "Smooth Nonparametric Estimation of the Distribution and Density Functions from Record-breaking Data." Communications in Statistics-Theory and Methods, 23, 1259-1274.

Gulati S, Padgett WJ (1994b). "Smooth Nonparametric Estimation of the Hazard and Hazard Rate Functions from Record-breaking Data." Journal of Statistical Planning and Inference, 42, 331-341.

Gulati S, Padgett WJ (1995). "Nonparametric Function Estimation from Inversely Sampled Record-breaking Data." Canadian Journal of Statistics, 23, 359-368.

Habibi A, Arghami NR, Ahmadi J (2006). "Statistical Evidence in Experiments and in Record Values." Communications in Statistics-Theory and Methods, 35, 1971-1983.

Johnson NL, Kotz S (1970). Distributions in Statistics-Continuous Univariate Distributons 1-2. John Wiley \& Sons.

Khan RU, Zia B (2009). "Recurrence Relations for Single and Product Moments of Record Values from Gompertz Distribution and a Characterization." World Applied Sciences Journal, 7, 1331-1334.

Kibria BMG (2004). "Performance of the Shrinkage Preliminary Test Ridge Regression Estimators Based on the Conflicting of W, LR and LM Tests." Journal of Statistical Computation and Simulation, 74, 793-810.

Kibria BMG, Saleh AKME (1993). "Performance of Shrinkage Preliminary Test Estimator in Regression Analysis." Jahangirnagar Review, A, 17, 133-148.

Kibria BMG, Saleh AKME (2004). "Preliminary Test Ridge Regression Estimators with StudentâĂŹs t Errors and Conflicting Test-statistics." Metrika, 59, 105-124.

Kibria BMG, Saleh AKME (2005). "Comparison between Han-Bancroft and Brook Methods to Determine the Optimal Significance Level for Pre-test Estimator." Journal of Probability and Statistical Science, 3, 293-303. 
Kibria BMG, Saleh AKME (2006). "Optimum Critical Value for Pre-test Estimator." Communications in Statistics-Simulation and Computation, 35, 309-319.

Kibria BMG, Saleh AKME (2010). "Preliminary Test Estimation of the Parameters of Exponential and Pareto Distributions for Censored Samples." Statistical Papers, 51, 757-773.

Lai CD, Xie M, Murthy DNP (2003). "A Modified Weibull Distribution." IEEE Transactions on Reliability, 52, 33-37.

Lawless JF (1982). Statistical Models and Methods for Lifetime Data, volume 362. John Wiley \& Sons.

Ljubo M (1965). "Curves and Concentration Indices for Certain Generalized Pareto Distributions." Statistical Review, 15, 257-260.

Lomax KS (1954). "Business Failures: Another Example of the Analysis of Failure Data." Journal of the American Statistical Association, 49, 847-852.

Mahmoud MAW, Al-Nagar HSH (2009). "On Generalized Order Statistics from Linear Exponential Distribution and Its Characterization." Statistical Papers, 50, 407-418.

Moore AH, Bilikam JE (1978). "Bayesian Estimation of Parameters of Life Distributions and Reliability from Type II Censored Samples." IEEE Transactions on Reliability, 27, 64-67.

Nagaraja HN (1988a). "Record Values and Related Statistics-a Review." Communications in Statistics-Theory and Methods, 17, 2223-2238.

Nagaraja HN (1988b). "Some Characterizations of Continuous Distributions Based on Regressions of Adjacent Order Statistics and Record Values." Sankhy : The Indian Journal of Statistics, Series A, pp. 70-73.

Nikulin M, Haghighi F (2006). "A Chi-squared Test for the Generalized Power Weibull Family for the Head-and-neck Cancer Censored Data." Journal of Mathematical Sciences, 133, $1333-1341$.

Razmkhah M, Morabbi H, Ahmadi J (2012). "Comparing Two Sampling Schemes Based on Entropy of Record Statistics." Statistical Papers, 53, 95-106.

Saleh AKME (2006). Theory of Preliminary Test and Stein-Type Estimations with Applications, volume 517. John Wiley \& Sons.

Saleh AKME, Kibria BMG (1993). "Performance of Some New Preliminary Test Ridge Regression Estimators and Their Properties." Communications in Statistics-Theory and Methods, 22, 2747-2764.

Saleh AKME, Sen PK (1978). "Nonparametric Estimation of Location Parameter after a Preliminary Test on Regression." The Annals of Statistics, 6, 154-168.

Tadikamalla PR (1980). "A Look at the Burr and Related Distributions." International Statistical Review, pp. 337-344.

Watson RI (1952). "Research Design and Methodology in Evaluating the Results of Psychotherapy." Journal of Clinical Psychology, 8, 29-33. 


\title{
Affiliation:
}

\author{
Ajit Chaturvedi \\ Department of Statistics \\ University of Delhi \\ Delhi-110007, India \\ E-mail:ajitc2001@yahoo.co.in
}

Suk-Bok Kang

Department of Statistics

Yeungnam University

Gyeongsan 38541, Republic of Korea

Corresponding Author E-mail: sbkang@yu.ac.kr

\author{
Ananya Malhotra \\ Department of Statistics \\ University of Delhi \\ Delhi-110007, India \\ E-mail:malhotra.ananya3@gmail.com
}

\section{Austrian Journal of Statistics}

published by the Austrian Society of Statistics

Volume 48

July 2019 http://www.ajs.or.at/

http://www.osg.or.at/

Submitted: 2018-06-21

Accepted: 2018-11-15 